\title{
Observational signatures of past mass-exchange episodes in massive binaries: the case of LSS $3074 \star, \star \star$
}

\author{
F. Raucq ${ }^{1}$, E. Gosset ${ }^{1}$, G. Rauw ${ }^{1}$, J. Manfroid ${ }^{1}$, L. Mahy ${ }^{1}$, N. Mennekens ${ }^{2}$, and D. Vanbeveren ${ }^{2}$ \\ ${ }^{1}$ Space sciences, Technologies and Astrophysics Research (STAR) Institute, Liège University, Quartier Agora, Allée du 6 Août 19c, \\ Bât. B5c, 4000 Liège, Belgium \\ e-mail: fraucq@doct.ulg.ac.be \\ 2 Astronomy and Astrophysics Group, Vrije Universiteit Brussel, Pleinlaan 2, 1050 Brussels, Belgium
}

Received 22 December 2016 / Accepted 13 February 2017

\begin{abstract}
Context. The role of mass and momentum exchanges in close massive binaries is very important in the subsequent evolution of the components. Such exchanges produce several observational signatures such as asynchronous rotation and altered chemical compositions, that remain after the stars detach again.

Aims. We investigated these effects for the close O-star binary LSS 3074 (O4f + O6-7:(f):), which is a good candidate for a past Roche lobe overflow (RLOF) episode because of its very short orbital period, $P=2.185$ days, and the luminosity classes of both components.

Methods. We determined a new orbital solution for the system. We studied the photometric light curves to determine the inclination of the orbit and Roche lobe filling factors of both stars. Using phase-resolved spectroscopy, we performed the disentangling of the optical spectra of the two stars. We then analysed the reconstructed primary and secondary spectra with the CMFGEN model atmosphere code to determine stellar parameters, such as the effective temperatures and surface gravities, and to constrain the chemical composition of the components.

Results. We confirm the apparent low stellar masses and radii reported in previous studies. We also find a strong overabundance in nitrogen and a strong carbon and oxygen depletion in both primary and secondary atmospheres, together with a strong enrichment in helium of the primary star.

Conclusions. We propose several possible evolutionary pathways through a RLOF process to explain the current parameters of the system. We confirm that the system is apparently in overcontact configuration and has lost a significant portion of its mass to its surroundings. We suggest that some of the discrepancies between the spectroscopic and photometric properties of LSS 3074 could stem from the impact of a strong radiation pressure of the primary.
\end{abstract}

Key words. stars: early-type - stars: fundamental parameters - stars: massive - stars: individual: LSS 3074 - binaries: eclipsing binaries: spectroscopic

\section{Introduction}

Some recent studies have shown that a high percentage of massive stars belong to binary or higher multiplicity systems (e.g. Mason et al. 1998; Sana et al. 2012, 2014; Sota et al. 2014). This multiplicity enables us to observationally determine the minimum masses of the stars through their orbital motion, but it also influences the evolution of the stars in various ways (e.g. Langer 2012). These evolutionary effects range from tidally induced rotational mixing (e.g. de Mink et al. 2009), over exchange of

* Based on observations collected at the European Southern Observatory (La Silla, Chile) and the Cerro Tololo Inter-American Observatory (Chile). CTIO is a division of the National Optical Astronomy Observatory (NOAO). NOAO is operated by the Association of Universities for Research in Astronomy (AURA), Inc., under cooperative agreement with the National Science Foundation (USA). Also based on observations collected with XMM-Newton, an ESA science mission with instruments and contributions directly funded by ESA member states and the USA (NASA).

$\star \star$ Tables A.1-A.3 are only available at the CDS via anonymous ftp to cdsarc.u-strasbg. fr (130.79.128.5) or via

http://cdsarc.u-strasbg.fr/viz-bin/qcat?J/A+A/601/A133 matter and angular momentum through a Roche lobe overflow (RLOF) interaction (e.g. Podsiadlowski et al. 1992; de Loore \& Vanbeveren 1994; Wellstein et al. 2001; Hurley et al. 2002), to the merging of both stars (e.g. Podsiadlowski et al. 1992; Wellstein et al. 2001). In RLOF interactions, one distinguishes three different situations: case A, if the RLOF episode occurs when the mass donor is on the core hydrogen-burning main sequence; case $\mathrm{B}$, when the star is in the hydrogen shell burning phase; and case $\mathrm{C}$, when the star is in the helium shell burning phase (Kippenhahn \& Weigert 1967, Vanbeveren et al. 1998). Such binary interactions significantly affect the physical properties of the components and their subsequent evolution. Despite considerable progresses in theoretical models, a number of open issues, such as the actual efficiency of accretion, remain (e.g. Wellstein et al. 2001; de Mink et al. 2007; Dray \& Tout 2007). To better understand this phenomenon, in-depth studies of systems undergoing or having undergone mass exchange are needed.

In this context, the short-period spectroscopic binary LSS 3074 (also identified as V889 Cen and ALS 3074), classified as ${\mathrm{O} 4 \mathrm{f}^{+}}^{+}$O6-7:(f): (Morrell \& Niemela 1990), with $P_{\text {orb }}=2.185$ days, is an extremely interesting target. This system 
is located behind the Coalsack region. The distance of this region was evaluated as $188 \pm 4$ pc (Seidensticker 1989; and Seidensticker \& Schmidt-Kaler 1989). LSS 3074 harbours one of the very few known O4f stars. These objects are rare since they probably represent a short-lived transition phase in the evolution of massive O-type stars before they become Wolf-Rayet stars. In addition, the very short orbital period of this system makes it a good candidate for a past RLOF episode. Obtaining a good orbital and photometric solution for this system and determining the fundamental properties of its components are therefore of the utmost importance to better understand these transition objects.

A first preliminary orbital solution of LSS 3074 was presented by Morrell \& Niemela (1990). The low $m \sin ^{3} i$ values inferred from this orbital solution $\left(8\right.$ and $\left.9 M_{\odot}\right)$ are rather surprising for such early-type objects. Niemela et al. (1992) provided an improved orbital solution and they discussed the phaselocked polarization variability of this system. Fitting a model to these variations, they inferred an inclination of $i=75^{\circ}$, yielding very low absolute masses of 10-11 $M_{\odot}$ and 11-12 $M_{\odot}$ for the $\mathrm{O} \mathrm{f}^{+}$and the O6-7 component, respectively. Niemela et al. cautioned however that tidal deformations could introduce additional polarization, biasing the inclination towards $90^{\circ}$. Optical light variations of LSS 3074 were first reported by Haefner et al. (1994), although these authors did not achieve a phase coverage of the light curve allowing them to clearly distinguish ellipsoidal variations from photometric eclipses. Haefner et al. suggested an inclination of $50-55^{\circ}$, yielding again rather low masses of 17-21 $M_{\odot}$ for both components.

In the present study, we discuss our determination of the orbital solution of this system and of the fundamental parameters of its components through several analysis techniques. Some very preliminary results were given in Gosset et al. (2005). The data used in our study are discussed in Sect. 2. In Sect. 3 and Sect. 4, we present the optical spectrum of LSS 3074 and our determination of its orbital solution. In Sect. 5, we perform a line profile variability study of several important lines of the optical spectrum of LSS 3074. In Sect.6, we present the preparatory treatment of our data, including the disentangling of the observed spectra to reconstruct individual spectra of the binary components needed for the subsequent spectral analysis, and in Sect. 7, we analyse the light curve. The spectral analyses, carried out with the non-LTE model atmosphere code CMFGEN, are presented in Sect. 8. In Sect.9, we conclude by a discussion concerning the evolutionary status of LSS 3074.

\section{Observations and data reduction}

\subsection{Spectroscopy}

Optical spectra of LSS 3074 were gathered with different instruments during several observing runs between 2002 and 2004 (see Table 1).

Three spectra were obtained with the EMMI instrument on the New Technology Telescope (NTT) at the European Southern Observatory (ESO) at La Silla during a three-day observing run in March 2002. The EMMI instrument was used in the échelle mode with grating \#9 and grism \#3, providing a resolving power of 7700. The data were reduced using the ECHELLE context of MIDAS, and 16 usable spectral orders, covering the wavelength domain from 4040 to $7000 \AA$, were extracted and normalized individually.
Twelve échelle spectra of LSS 3074 were taken with the Fiber-fed Extended Range Optical Spectrograph (FEROS; Kaufer et al. 1999). In April 2002, the spectrograph was attached to the ESO $1.52 \mathrm{~m}$ telescope at La Silla, while in May 2003 and May 2004, it was used at the $2.2 \mathrm{~m} \mathrm{ESO/MPE}$ telescope at La Silla. The exposure times were 40-75 $\mathrm{min}$. The detector was an EEV CCD with $2048 \times 4096$ pixels of $15 \mu \mathrm{m} \times 15 \mu \mathrm{m}$. We used an improved version of the FEROS context within the MIDAS package provided by ESO to reduce the data (Sana et al. 2006a).

\subsection{Photometry}

During March-April-May 2001, LSS 3074 was observed in photometry with the Yale $1 \mathrm{~m}$ telescope at the Cerro Tololo Interamerican Observatory. The telescope was operated in service mode (project A01A0098, PI E. Gosset) by the YALO consortium. The telescope was equipped with the imaging camera ANDICAM.

Beyond some shortening due to bad weather conditions, the run was initially split into two periods: from HJD 2451992 (2001-03-24) to HJD 2452003 (2001-04-04) and from HJD 2452023 (2001-04-24) to HJD 2452037 (2001-05-08). It was designed to optimize the Fourier spectral window for period determination with the least observing effort, while reaching a frequency resolution corresponding to a total duration of about 50 days without being hampered by the aliasing due to the central gap. In order to reduce the effect of the one-day aliasing in the photometric data, we tried to observe the star three times per night roughly at $3 \mathrm{~h}$ before meridian, at meridian and at $3 \mathrm{~h}$ after it.

The ANDICAM camera was equipped with a Loral CCD of 2048 by $204815 \mu \mathrm{m}$ pixels. The projection of a pixel on the sky corresponds to $0.30^{\prime \prime}$ allowing a convenient sampling. The whole field was thus covering a $10^{\prime}$ by $10^{\prime}$ square on the sky. The CCD was read out with two amplifiers. At the time of the observations it was characterized by a $11 \mathrm{e}^{-} /$pixel readout noise; the gain was set to $3.6 \mathrm{e}^{-}$per ADU. Half of the CCD presented a variable noise pattern but the amplitude was sufficiently small not to be a concern. Moreover, LSS 3074 was systematically centred on the best half of the CCD.

The various measurements were performed using the Johnson system $B, V, R$, and $I$ filters. Each measurement consisted of exposures of $2 \times 10 \mathrm{~s}$ in $B, 2 \times 3 \mathrm{~s}$ in $V, 1 \times 1 \mathrm{~s}$ in $R$, and $2 \times 1 \mathrm{~s}$ in $I$. For each night, a minimum of five dome flat fields in each filter and of 20 bias frames were acquired. A few Stetson standard fields (PG0918, PG1047, PG1323, PG1633, and SA107) were observed on three good nights (May 4, 5, and 8) to calibrate the above-mentioned photometric observations.

We reduced the raw data in the standard way (bias subtraction, division by a combined flat field, etc.) independently for the two halves of the CCD. The list of objects were built and the relevant instrumental magnitudes were extracted via DAOPHOT (Stetson 1987) under IRAF with both an aperture integration approach and a psf-fitting technique. A consistent natural system was established using a multi-night, multi-star, and multi-filter method as described in Manfroid (1993; see also Manfroid et al. 2001). A set of constant stars was iteratively constructed to perform precise relative photometry. The differential data were calculated with an aperture radius of $1.5^{\prime \prime}$. A least-square fit of the standard star data allowed us to obtain absolute zero points and colour transformation coefficients using the large aperture $\left(3.3^{\prime \prime}\right)$ 
Table 1. Journal of the spectroscopic observations of LSS 3074.

\begin{tabular}{cccccccc}
\hline \hline HJD-2 450 000 & Instrument & $\begin{array}{c}\text { Exp. time } \\
(\mathrm{min} .)\end{array}$ & $\phi$ & $\begin{array}{c}\mathrm{RV}_{1} \\
\left(\mathrm{~km} \mathrm{~s}^{-1}\right)\end{array}$ & $\begin{array}{c}\mathrm{RV}_{2} \\
\left(\mathrm{~km} \mathrm{~s}^{-1}\right)\end{array}$ & $\begin{array}{c}\mathrm{RV}_{1, \text { corr }} \\
\left(\mathrm{km} \mathrm{s}^{-1}\right)\end{array}$ & $\begin{array}{c}\mathrm{RV}_{2, \text { corr }} \\
\left(\mathrm{km} \mathrm{s}^{-1}\right)\end{array}$ \\
\hline 2353.719 & EMMI & 60 & 0.48 & -68.9 & & -70.3 & \\
2354.691 & EMMI & 60 & 0.93 & -183.6 & 72.4 & -184.2 & 78.6 \\
2355.720 & EMMI & 60 & 0.40 & 108.3 & $-160.7:$ & 101.2 & $-154.4:$ \\
2381.596 & FEROS & 60 & 0.24 & 165.8 & -203.6 & 161.9 & -197.4 \\
2382.597 & FEROS & 60 & 0.70 & $-246.7:$ & $170.8:$ & $-251.8:$ & $159.1:$ \\
2383.598 & FEROS & 60 & 0.15 & $127.9:$ & $-178.9:$ & $123.2:$ & $-172.6:$ \\
2783.576 & FEROS & 60 & 0.19 & $169.9:$ & -192.9 & $165.2:$ & -196.7 \\
2784.620 & FEROS & 60 & 0.67 & $-255.6:$ & $177.8:$ & $-256.1:$ & $173.8:$ \\
3130.613 & FEROS & 60 & 0.01 & -51.3 & & -51.8 & \\
3131.594 & FEROS & 75 & 0.46 & -31.8 & & -29.1 & \\
3132.576 & FEROS & 75 & 0.90 & -198.0 & $77.3:$ & -203.1 & $69.1:$ \\
3133.629 & FEROS & 40 & 0.39 & 81.5 & -156.3 & 76.9 & -161.3 \\
3133.660 & FEROS & 40 & 0.40 & 68.5 & $-151.3:$ & 63.8 & $-156.3:$ \\
3134.578 & FEROS & 40 & 0.82 & -266.7 & 154.7 & -267.0 & 151.3 \\
3134.608 & FEROS & 40 & 0.83 & -257.8 & 162.8 & -258.3 & 160.8 \\
\hline
\end{tabular}

Notes. The phases $(\phi)$ are computed according to the ephemerides listed in Table 5. The radial velocities are presented in Sect. 4. The typical uncertainties on the RVs are $10-15 \mathrm{~km} \mathrm{~s}^{-1}$. The colons indicate uncertainties larger than $20 \mathrm{~km} \mathrm{~s}^{-1}$.

data. The derived colour transformation equations were

$$
\begin{gathered}
(B-V)_{\text {std }}=0.0236( \pm 0.0255) \\
+0.9991( \pm 0.0323)(B-V)_{\text {ctio }} \\
(V-R)_{\text {std }}=0.1016( \pm 0.0153) \\
+0.7804( \pm 0.0316)(V-R)_{\text {ctio }} \\
(V-I)_{\text {std }}=0.0404( \pm 0.0237) \\
+0.9396( \pm 0.0249)(V-I)_{\text {ctio }} \\
V_{\text {std }}=-0.0145( \pm 0.0181) \\
-0.0219( \pm 0.0229)(B-V)_{\text {ctio }}+V_{\text {ctio }}
\end{gathered}
$$

The internal precision of the data corresponds to $\sigma=0.007$, $0.010,0.013$, and 0.013 mag for the $B, V, R$, and $I$ filters respectively, whereas the accuracy of the absolute magnitudes turned out to be $\sigma=0.02 \mathrm{mag}$. The measurements for LSS 3074 are available at the CDS in Table A.1. During the iterative removing of the variable stars, two stars were rejected early in the process and turned out to be significantly variable, i.e. HD 116827 and LSS 3072. They are further discussed in Appendix A and the corresponding measurements are available at the CDS in Tables A.2 and A.3.

\subsection{XMM-Newton observations}

XMM-Newton (Jansen et al. 2001) observed LSS 3074 twice. The first observation (ObsID 0109100201), taken in August 2001, was centred on LSS 3074 itself. The three EPIC (Strüder et al. 2001; Turner et al. 2001) cameras were operated in full-frame mode and the medium filter was used to discard optical and UV light. The corresponding X-ray image of the field can be found in Gosset et al. (2005). A second observation (ObsID 0036140201), centred on the low-mass X-ray binary XB 1323-619, was taken in January 2003. This time, the EPICpn camera and the central chip of the EPIC-MOS1 were operated in timing mode, whilst the EPIC-MOS2 was used in full-frame mode. All EPIC cameras were used in combination with the thin filter. The detectors used in timing mode do not provide data
Table 2. Journal of X-ray observations of LSS 3074.

\begin{tabular}{ccccc}
\hline \hline Rev. & Instrument & $\begin{array}{c}\text { Duration } \\
(\mathrm{ks})\end{array}$ & $\begin{array}{c}\text { JD (start) } \\
-2450000\end{array}$ & $\begin{array}{c}\text { JD (end) } \\
-2450000\end{array}$ \\
\hline 0309 & MOS1 \& pn & $9.9 \& 6.0$ & 2138.830 & 2138.945 \\
0575 & MOS1 \& MOS2 & $50.5 \& 50.8$ & 2668.865 & 2669.452 \\
\hline
\end{tabular}

concerning LSS 3074. But since the star is located relatively far off axis in the second observation, it fell on one of the peripheral CCD chips of both MOS detectors. The raw data were processed with SAS v15.0.0 using calibration files available in June 2016 and following the recommendations of the XMM-Newton team $^{1}$. We notably filtered the data to keep only best-quality data (PATTERN of 0-12 for EPIC-MOS and 0-4 for EPIC-pn data). Whilst the background level was high during the first observation, no genuine background flares due to soft protons affected either of the two observations. In the first observation, LSS 3074 falls very close to the out-of-time events of the very bright X-ray binary in the MOS2 data, thus rendering these specific data difficult to use.

We extracted the EPIC spectra of LSS 3074 via the task especget. For the source regions, we used a circular region with radius 30", centred on the Simbad coordinates of the binary. The background was evaluated over an annulus with inner and outer radii of 30 and $45^{\prime \prime}$, respectively. Specific ARF and RMF response files were computed to calibrate the flux and energy axes. The EPIC spectra were grouped with the SAS command specgroup to obtain an oversampling factor of five and to ensure that a minimum signal-to-noise ratio of 3 (i.e. a minimum of 10 counts) was reached in each spectral bin of the backgroundcorrected spectra.

The EPIC spectrum of LSS 3074 peaks between 1.0 and $2.0 \mathrm{keV}$. It contains very few photons at energies below $1.0 \mathrm{keV}$ (mainly as a result of the heavy absorption) and displays very weak emission at energies above $2 \mathrm{keV}$.

\footnotetext{
1 http://xmm. esac. esa.int/sas/current/documentation/ threads/
} 
Table 3. X-ray spectral fits of LSS 3074.

\begin{tabular}{ccccccc}
\hline \hline Rev. & $\begin{array}{c}\log N_{\text {wind }} \\
\left(\mathrm{cm}^{-2}\right)\end{array}$ & $\begin{array}{c}k T \\
(\mathrm{keV})\end{array}$ & $\begin{array}{c}\text { Norm } \\
\left(\mathrm{cm}^{-5}\right)\end{array}$ & $\chi_{v}^{2}$ & $\begin{array}{c}f_{\mathrm{X}} \\
\left(10^{-14} \mathrm{erg} \mathrm{cm}^{-2} \mathrm{~s}^{-1}\right)\end{array}$ \\
\hline 0309 & $21.83_{-.48}^{+.25}$ & $0.99_{-.33}^{+.65}$ & $\left(1.26_{-0.57}^{+1.06}\right) 10^{-4}$ & $1.55(7)$ & 3.6 & 8.7 \\
0575 & $22.07_{-.16}^{+.18}$ & $0.47_{-.18}^{+.17}$ & $\left(4.72_{-2.54}^{+24.08}\right) 10^{-4}$ & $1.55(14)$ & 2.6 & 8.3 \\
comb. & $22.00_{-.16}^{+.15}$ & $0.57_{-.14}^{+.21}$ & $\left(2.61_{-1.03}^{+2.38}\right) 10^{-4}$ & $1.64(24)$ & 2.9 & 8.6 \\
\hline
\end{tabular}

Notes. The normalization of the apec models is given as $\frac{10^{-14} \int n_{\mathrm{e}} n_{\mathrm{H}} \mathrm{d} V}{d^{2}}$ where $d$ is the distance of the source (in cm), $n_{\mathrm{e}}$ and $n_{\mathrm{H}}$ are the electron and hydrogen densities of the source $\left(\right.$ in $\mathrm{cm}^{-3}$ ). The error bars indicate $1 \sigma$ uncertainties.

The fits of the X-ray spectra were performed with xspec (Arnaud 1996) version 12.9.0i. To evaluate the neutral hydrogen column density due to the interstellar medium (ISM), we adopt $B-V=1.44$. Accounting for the intrinsic $(B-V)_{0}$ quoted by Martins \& Plez (2006) and using the conversion between colour excess and neutral hydrogen column density of Bohlin et al. (1978), we estimate $N_{\mathrm{H}}=0.99 \times 10^{22} \mathrm{~cm}^{-2}$. The $\mathrm{X}$-ray absorption by the ISM was modelled using the TübingenBoulder model (Wilms et al. 2000). X-ray spectra from massive stars can be further absorbed by the material of the ionized stellar wind. To model such an absorption, we imported the stellar wind absorption model of Nazé et al. (2004) into xspec as a multiplicative tabular model (hereafter labelled as wind). To the first approximation, the emission from massive stars can be represented by models of collisionally ionized equilibrium optically thin thermal plasmas. In our fits, we used apec models (Smith \& Brickhouse 2001) computed with ATOMBD v2.0.2 as provided within xspec. The plasma abundances were taken to be solar (Asplund et al. 2009). Given the rather low quality of the spectra, reasonable fits were obtained for models of the kind tbabs $\times$ wind $\times$ apec. The results are listed in Table 3 . Whilst the best-fit parameters of the two observations differ, these differences could be due to the ambiguity between a soft, highly absorbed plasma and a harder, less absorbed plasma. We have thus also performed a fit of the combined datasets. The results are again quoted in Table 3 .

We find that the X-ray flux corrected for the ISM absorption is close to $8.6 \times 10^{-14} \mathrm{erg} \mathrm{cm}^{-2} \mathrm{~s}^{-1}$. If we consider the $B$ and $V$ magnitudes of LSS 3074 along with the bolometric corrections from Martins \& Plez (2006), we obtain $\log L_{\mathrm{X}} / L_{\mathrm{bol}} \simeq-7.3$ which, given the uncertainties on both the X-ray and bolometric fluxes, is entirely compatible with the canonical value observed for most O-type stars (Sana et al. 2006b; Nazé 2009, and references therein).

\section{Optical spectrum of LSS 3074}

The spectrum of LSS 3074 is illustrated in Fig. 1. The stellar spectrum displays many absorption lines of H I, He I, He II, $\mathrm{N}$ III, N IV, and N V. The most prominent emission lines are He II $\lambda$ 4686, N III $\lambda \lambda$ 4634-41, H $\alpha$, and He I $\lambda$ 5876. Weaker emission lines of Si IV $\lambda \lambda 4089,4116,6668$, and N IV $\lambda \lambda 4058,6212-20$ as well as P-Cygni emission components in He II $\lambda 5412$ and $\mathrm{H} \beta$ lines are also seen. In addition to some interstellar absorption lines $\left(\mathrm{Na}, \mathrm{CH}, \mathrm{CH}^{+}\right)$, there are also numerous (and rather strong) diffuse interstellar bands (DIBs; see Herbig 1995). The strength of these features is an indication of the heavy interstellar absorption towards LSS 3074.
Table 4. Adopted wavelengths of the lines used to measure the RVs of LSS 3074 .

\begin{tabular}{cc}
\hline \hline Spectrum & Wavelength $(\AA)$ \\
\hline H I & 4340.47 \\
He I & 4471.48 \\
He II & 4541.59 \\
He II & 5411.52 \\
He I & 5875.62 \\
He II & 6406.44 \\
He I & 7065.24 \\
\hline \multicolumn{2}{|}{} \\
\hline \hline Spectrum & Wavelength $(\AA)$ \\
\hline N IV & 4057.80 \\
N V & 4603.83 \\
N v & 4619.90 \\
N III & 4634.16 \\
N III & 4640.64 \\
\hline
\end{tabular}

Notes. These effective wavelengths are taken from Underhill (1995). The top part displays the lines we used for the determination of the orbital solution of the system. The bottom part shows the main $\mathrm{N}$ emission and absorption lines.

The presence of $\mathrm{NV} \lambda \lambda 4604$ and 4620 may indicate that one of the components of LSS 3074 is rather hot (O3-O4, Walborn 2001, Walborn et al. 2002). The radial velocities of the $\mathrm{N}$ III $\lambda \lambda 4634,4641$, and $\mathrm{N}$ IV $\lambda 4058$ emission lines and the $\mathrm{Nv}$ absorption lines are shown in Fig. 3 below and analysed in Sect. 6.2.

Several emission lines in the spectrum of the system display strong line profile variations. The most prominent modulations are seen in the He II $\lambda 4686$, He I $\lambda$ 5876, and $\mathrm{H} \alpha$ lines (see Fig. 4) and they are presented in Sect. 5 .

\section{Orbital solution}

Since LSS 3074 is a rather faint and heavily reddened object, the accuracy of any radial velocity (RV) determination is mainly limited by the $\mathrm{S} / \mathrm{N}$ ratio of the data. Therefore, we have concentrated our efforts on the strongest absorption lines that are essentially free from blends with other features. In this way, we have measured the RVs of $\mathrm{H} \gamma$, He I $\lambda \lambda 4471,5876$, and 7065 and He II $\lambda \lambda 4542,5412$, and 6406 in the spectra. We adopted the effective wavelengths of Underhill (1995), as listed in Table 4.

For each observation, the RVs of the primary and secondary components were computed as the mean of the corresponding 
F. Raucq et al.: Signatures of past mass exchange in LSS 3074
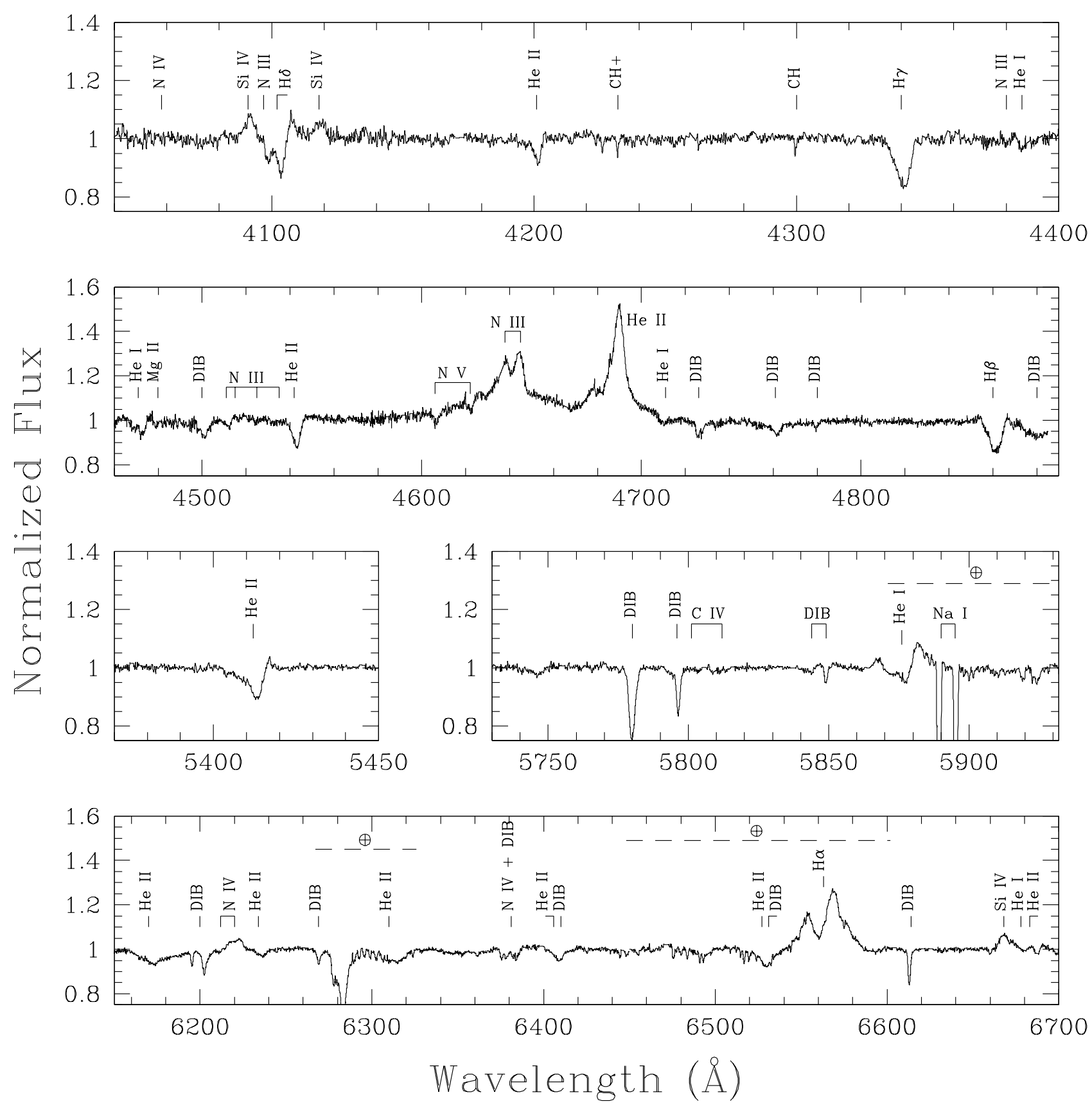

Fig. 1. Most important parts of the optical spectrum of LSS 3074 as observed with the EMMI instrument at the NTT on HJD $2452355.7, \phi=0.40$. This spectrum is not corrected for the telluric absorption, and the main telluric bands are shown with dashed lines (Curcio et al. 1964).

RVs measured for the above listed lines on that observation. Unfortunately, either because of a low S/N or other problems, not all of these lines could be measured on all of our spectra. For instance, while we managed to deblend the primary and secondary components of the He I lines on most of our spectra, the intensity contrast between the two components is much larger for $\mathrm{H} \gamma$ and for the He II lines. Therefore, the secondary component of the latter lines could only be resolved on the spectra with the highest $\mathrm{S} / \mathrm{N}$ ratio. Another example is $\mathrm{He}$ II $\lambda 5412$ : the proximity of several DIBs of similar strength as the secondary line and the fact that the line develops a P-Cygni type profile (with an emission component at a velocity of $\sim 200 \mathrm{~km} \mathrm{~s}^{-1}$ ) between phase $\phi=0.35$ and 0.50 lead to rather complex blends and thus highly uncertain RVs at these specific orbital phases. As a result, not all of the RV data points are based on the same set of lines. Yet, it is well known that in early-type stars featuring relatively strong winds, the systemic velocities of the different spectral lines can be significantly different (Rauw et al. 2002). Therefore combining different sets of spectral lines for different dates could bias the orbital solution. To circumvent this problem, we tied the RVs of the different lines to a single systemic velocity for each component. To do so, we first noted that the He I $\lambda 5876$ line is the one for which RVs of both components could be determined on nearly all observations, despite the fact that these absorptions lie on top of a broader emission. We thus selected He I $\lambda 5876$ as our reference line. The positions of the primary and secondary He I $\lambda 5876$ lines are shown in Fig. 4. For each of the other lines that we measured, we then determined the mean RV shift compared to He I $\lambda 5876$, and we subtracted this mean shift from the actual measurements. For each observation, the corrected RVs 
were then averaged, resulting in a homogeneous set of values, expressed in the rest frame of the He I $\lambda 5876$ line.

These resulting RVs are listed in Table 1. For most data points, the uncertainties on these RVs are about $10-15 \mathrm{~km} \mathrm{~s}^{-1}$. In some cases however (indicated by the colons in Table 1), the uncertainties are larger than $20 \mathrm{~km} \mathrm{~s}^{-1}$ and can reach $\sim 40 \mathrm{~km} \mathrm{~s}^{-1}$ in one case (HJD 2452 382.597). To adopt the same notations as previous investigators, we refer to the brightest and hottest component of LSS 3074 as the primary, though its present-day mass appears to be lower than that of its companion (see below).

Owing to the severe aliasing problem, it is extremely difficult to obtain an independent determination of the orbital period of LSS 3074 from our set of RV measurements only. A Fourier analysis of the $\mathrm{RV}_{1}$ and $\mathrm{RV}_{2}$ data yields the highest peaks around $v=0.46020 \mathrm{~d}^{-1}\left(P_{\text {orb }}=2.1730\right.$ days $)$ and $v=0.45762 \mathrm{~d}^{-1}$ $\left(P_{\text {orb }}=2.1852\right.$ days $)$. However, we caution that there are many more aliases that could hide the actual orbital period. It is worth pointing out that the second peak yields almost exactly the same period as found by Niemela et al. (1992). We performed the same Fourier analysis for each of the four bandpasses of the photometric data, $B, V, R$, and $I$, and obtained a highest peak at $v=0.9158 \mathrm{~d}^{-1}$, which corresponds to an orbital frequency of $v=0.9158 / 2=0.4579 \mathrm{~d}^{-1}$, corresponding to $P_{\text {orb }}=2.184$ days. While the periodogram of the photometric time series allows us to unambiguously identify the correct alias, the natural width of its peaks is much larger than in the case of our spectroscopic time series. In fact, folding the RVs into the photometric period yields an unacceptably large scatter. Therefore, combining the results from periodograms of the photometric and spectroscopic time series, we find that the most likely period of LSS 3074 is $2.1852 \pm 0.0006$ days, with the error bars given as $1 \sigma$ deviation.

Also, the $T_{0}$ obtained with the photometric curves is HJD 2452000.9568 , which corresponds to a phase shift of $\sim 0.05$ with the $T_{0}$ obtained with the RV data. If we lock the RV data to this $T_{0}$, we obtain a period of 2.1849 days. Considering that this result is well within the error bars of our previous calculations, we can admit the phase alignment of the RV and photometric data.

As a consistency check, we also combined our measurements in the $V$ band with those taken by Haefner et al. (1994) and obtained a highest peak of the periodogram at $v=0.9153 \mathrm{~d}^{-1}$, which corresponds to an orbital frequency of $v=0.9153 / 2=$ $0.45765 \mathrm{~d}^{-1}$, corresponding to $P_{\text {orb }}=2.1851$ days. This result tends to confirm the quality of our determination of the orbital period of the system.

Adopting an orbital period of 2.1852 days, we computed an orbital solution assuming a circular orbit. The RVs were weighted according to their estimated uncertainties. The result is shown in Fig. 2 and the corresponding orbital elements are provided in Table 5.

The RV amplitudes of our solution are in reasonable agreement with values from the literature $\left(K_{1}=222\right.$ and $K_{2}=$ $218 \mathrm{~km} \mathrm{~s}^{-1}$; Niemela et al. 1992). Consequently our orbital solution also leads to low minimum masses, similar to those of Niemela et al. ( $m \sin ^{3} i=9.5$ and $10 M_{\odot}$ for the O4f primary and the secondary, respectively). The apparent systemic velocity of the primary is significantly more negative than that of the secondary. This most likely indicates that the absorption lines of the primary are not entirely formed in the static photosphere, but arise at least partly from an expanding stellar wind.

Figure 3 shows the RVs of the nitrogen emission and absorption lines in the spectrum of LSS 3074. The adopted effective wavelengths of the studied lines are listed in Table 4 . All the lines seem to move with the primary, but the scatter of the velocities
Table 5. Orbital solution computed from our RV data of LSS 3074 assuming a circular orbit and an orbital period of 2.1852 days.

\begin{tabular}{lcc}
\hline \hline & Primary & Secondary \\
\hline$T_{0}(\mathrm{HJD}-2450000)$ & \multicolumn{2}{c}{$2000.851 \pm 0.008$} \\
$\gamma\left(\mathrm{km} \mathrm{s}^{-1}\right)$ & $-66.0 \pm 5.0$ & $-21.7 \pm 4.7$ \\
$K\left(\mathrm{~km} \mathrm{~s}^{-1}\right)$ & $228.5 \pm 7.1$ & $196.0 \pm 6.1$ \\
$a \sin i\left(R_{\odot}\right)$ & $9.9 \pm 0.3$ & $8.5 \pm 0.3$ \\
$q=m_{1} / m_{2}$ & \multicolumn{2}{c}{$0.86 \pm 0.04$} \\
$m \sin ^{3} i\left(M_{\odot}\right)$ & $8.0 \pm 0.5$ & $9.3 \pm 0.7$ \\
$R_{\mathrm{RL}} /\left(a_{1}+a_{2}\right)$ & $0.37 \pm 0.01$ & $0.39 \pm 0.01$ \\
$R_{\mathrm{RL}} \sin i\left(R_{\odot}\right)$ & $6.7 \pm 0.2$ & $7.2 \pm 0.2$ \\
$\sigma_{\text {fit }}$ & \multicolumn{2}{c}{3.11} \\
\hline
\end{tabular}

Notes. $T_{0}$ refers to the time of conjunction with the primary being in front. $\gamma, K$ and $a \sin i$ denote respectively the apparent systemic velocity, the semi-amplitude of the radial velocity curve and the projected separation between the centre of the star and the centre of mass of the binary system. $R_{\mathrm{RL}}$ stands for the radius of a sphere with a volume equal to that of the Roche lobe computed according to the formula of Eggleton (1983). All error bars indicate $1 \sigma$ uncertainties.

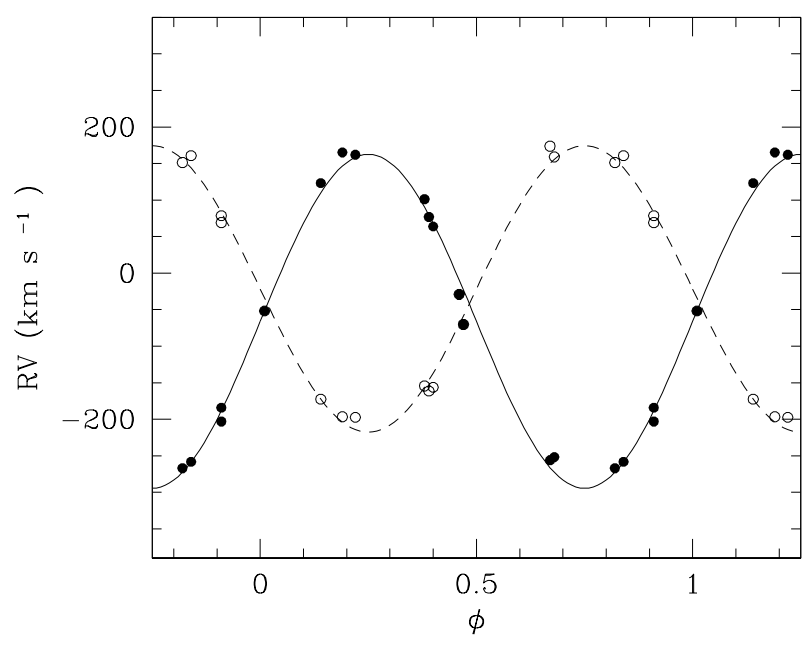

Fig. 2. Radial velocities of the components of LSS 3074 assuming a period of 2.1852 days. The RVs of the primary and secondary stars are shown with filled and empty circles, respectively. The solid and dashed lines indicate the orbital solution from Table 5.

of the various lines is rather large, especially at phases between the 0.25 quadrature and 0.5 conjunction. During this phase interval, the N III emission lines yield systematically larger velocities than the $\mathrm{N} \mathrm{V}$ absorption.

\section{Line profile variability}

The most prominent emission lines in the optical spectrum of LSS 3074 show strong profile variations (see Fig. 4). For instance, the He II $\lambda 4686$ line evolves from a broad and skewed emission (around $\phi=0.0$ ) into a double-peaked feature with rather narrow individual peaks and the strongest peak closely following the orbital motion of the primary (at phases near 0.5 ).

On the other hand, the $\mathrm{H} \alpha$ emission features a double-peaked profile on most of our spectra and the profiles observed around the two conjunctions are very similar. At phases near quadrature, the peaks are broader and less clear cut. Apart from the moving absorption lines, the He I $\lambda 5876$ profile has a morphology relatively similar to that of the $\mathrm{H} \alpha$ line. 


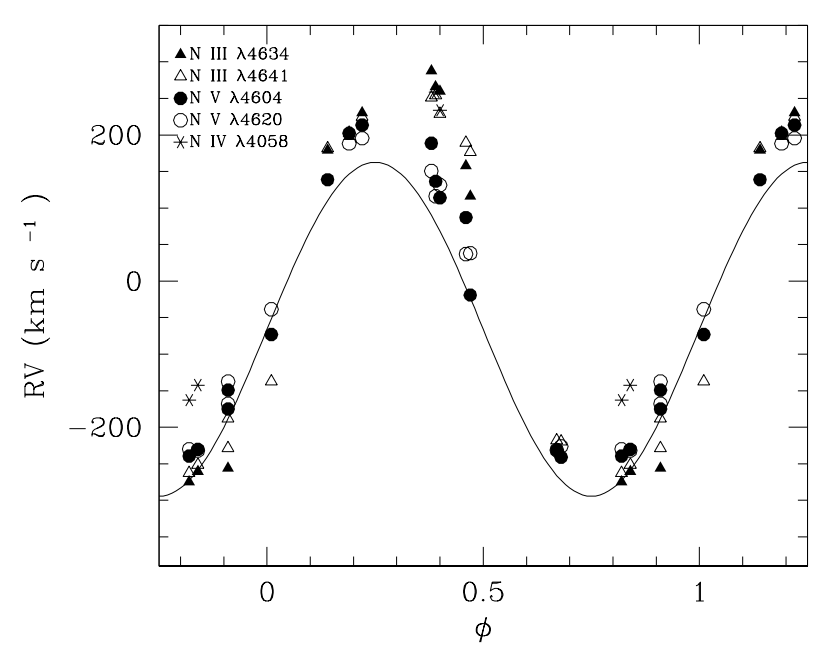

Fig. 3. Radial velocities of the N III and N IV emission lines and the N v absorptions in the spectrum of LSS 3074. The meaning of the various symbols is given in the upper left corner. The solid line yields the orbital solution of the primary from Table 5 .

We measured the equivalent widths (EWs) of the $\mathrm{H} \alpha$ line between 6513 and $6604 \AA$. We find that $\overline{E W}=4.09 \pm 0.60 \AA$. Although the dispersion around the mean is quite large, no obvious phase-locked behaviour is apparent. In particular, we do not see any variations attributable to the modulation of the continuum.

These complex line morphologies make it impossible to assign a single RV to the He II $\lambda 4686$ and $\mathrm{H} \alpha$ emission lines, and suggest that these lines do not arise in the atmosphere of one of the two stars, but stem from an extended emission region. To further quantify this situation, we used the method of Doppler tomography to map the emitting regions of the He II $\lambda 4686$ and $\mathrm{H} \alpha$ lines in velocity space. Our method is based on the Fourier-filtered back projection technique (Horne 1991; Rauw et al. 2002; Mahy 2011). The radial velocity of any gas flow that is stationary in the rotating frame of reference of the binary can be expressed as

$v(\phi)=v_{x} \cos (2 \pi \phi)-v_{y} \sin (2 \pi \phi)+v_{z}$

where $\phi$ stands for the orbital phase with $\phi=0.0$, corresponding to the primary star being in front. The $\left(v_{x}, v_{y}, v_{z}\right)$ are the velocity coordinates of the gas flow. The $x$ and $y$ axes are located in the orbital plane. The $x$-axis runs from the primary to the secondary, whilst the positive $y$-axis points in the direction of the orbital motion of the secondary. The $v_{z}$ component represents the apparent systemic velocity of the line under consideration. The Doppler map consists of a projection of relation given by (5) on the $\left(v_{x}, v_{y}\right)$ plane. For a given value of $v_{z}$, each pixel in a Doppler map, specified by its velocity coordinates, is therefore associated with a particular form of Eq. (5). All our spectra listed in Table 1 were given equal weights. The Doppler maps, computed adopting $v_{z}=-44 \mathrm{~km} \mathrm{~s}^{-1}$, are shown in Fig. 5. They reveal extended line formation regions in velocity space with different morphologies for the two lines. The He II $\lambda 4686$ line features an emission lobe on the negative $v_{x}$ side and mostly with negative $v_{y}$. This emission seems thus mostly associated with the primary star. The $\mathrm{H} \alpha$ Doppler map displays two lobes, extending on both sides of the Roche lobes in velocity space, with the strongest one closely matching the emission lobe of the Doppler map of He II $\lambda 4686$.

A full interpretation of these Doppler maps in terms of windwind interactions is beyond the scope of the present paper. However, it is interesting to compare our Doppler maps with those of Algol-type systems where a cool star fills up its Roche lobe and transfers matter to a hotter companion (Richards et al. 2014). Our maps are clearly different from those presented by Richards et al. (2014). The structures seen in Fig. 5 neither resemble those produced by gas streams that directly impact the mass gainer nor do they feature the ring-like structure, centred on the mass gainer, that appears for systems with Keplerian accretion disks. These results therefore do not support the presence of an accretion disk or an accretion stream in LSS 3074.

\section{Preparatory analysis}

\subsection{Spectral disentangling}

The determination of the orbital solution allowed us to recover the individual spectra of both components through the disentangling of the normalized spectra of the binary system. For this purpose, we used our disentangling routine (Rauw 2007) based on the method of González \& Levato (2006), previously used by Linder et al. (2008) and improved by Mahy et al. (2012). In this procedure, the mean spectra of each binary component are reconstructed in an iterative way by shifting the observed data into the frame of reference of one star and subtracting the best approximation of the spectrum of the other star shifted to its observed radial velocity. In the disentangling carried out in this work, we fixed the RVs of the binary components to those corresponding to the orbital solution of Sect. 4. For a more detailed description of the method, see Raucq et al. (2016).

As for any disentangling method, this technique also has its limitations (González \& Levato 2006). An important limitation for our study is that broad spectral features are not recovered with the same accuracy as narrow ones. Indeed, features that are wider than a few times the RV amplitude are barely properly recovered. This is the case of the wings of the Balmer lines, for example. Moreover small residual errors in the normalization of the input spectra can lead to oscillations of the continuum in the resulting disentangled spectra on wavelength scales of several dozen $\AA$, and a reasonable observational sampling of the orbital cycle is needed because the quality of the results depends on the radial velocity ranges covered by the observations. Finally, spectral disentangling works on continuum-normalized spectra and does not yield the brightness ratio of the stars, which must be determined by other techniques (see below).

In the particular case of LSS 3074, we also encountered problems due to the large brightness ratio of the system. Indeed, as shown in Sect. 6.3, the primary star appears much brighter than the secondary star, meaning that the latter is faint in the observed spectra, which induces difficulties in the disentangling procedure, a lower $\mathrm{S} / \mathrm{N}$ ratio, and thus larger uncertainties on the resulting secondary spectrum.

\subsection{Spectral types}

We then determined the spectral types of the stars through the measurement of the equivalent width ratio of some spectral lines of the reconstructed individual line spectra of the primary and secondary components. We used the He I $\lambda 4471$ and He II $\lambda 4542$ lines, on the one hand, and Si IV $\lambda 4089$ and He I $\lambda 4143$ lines, on the other hand, and applied the Conti quantitative classification criteria for O-type stars (Conti \& Alschuler 1971; Conti \& Frost 1977; see also van der Hucht 1996) for both spectral types and luminosity classes. In this way, we obtain O5.5 I and O6.5-7 I classifications for the primary and secondary, respectively. The strong He II $\lambda 4686$ and N III $\lambda \lambda$ 4634-41 emission 


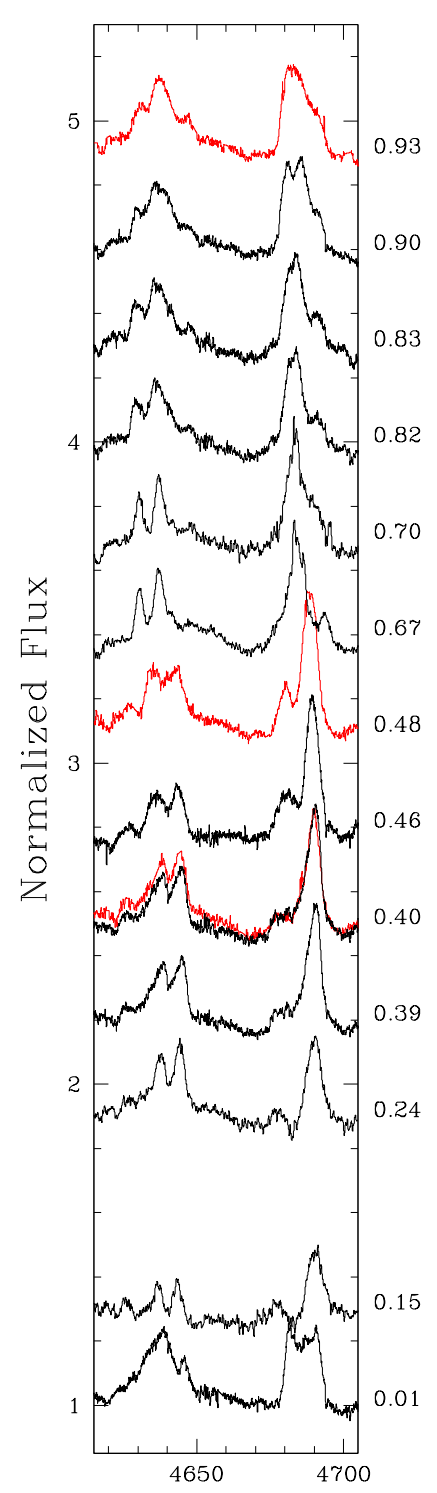

Wavelength $(\AA)$

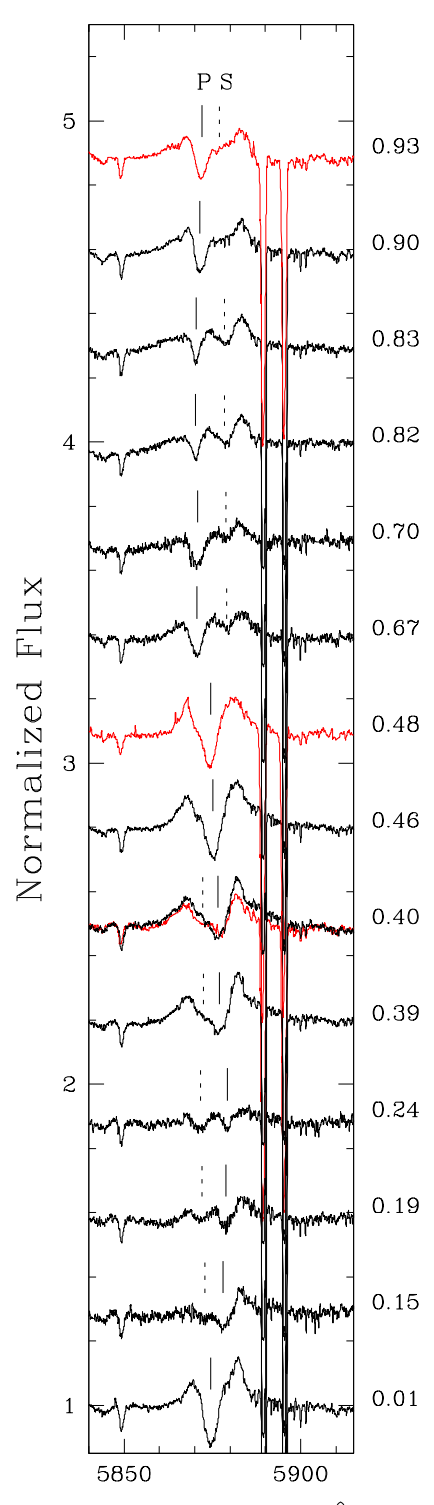

Wavelength $(\AA)$

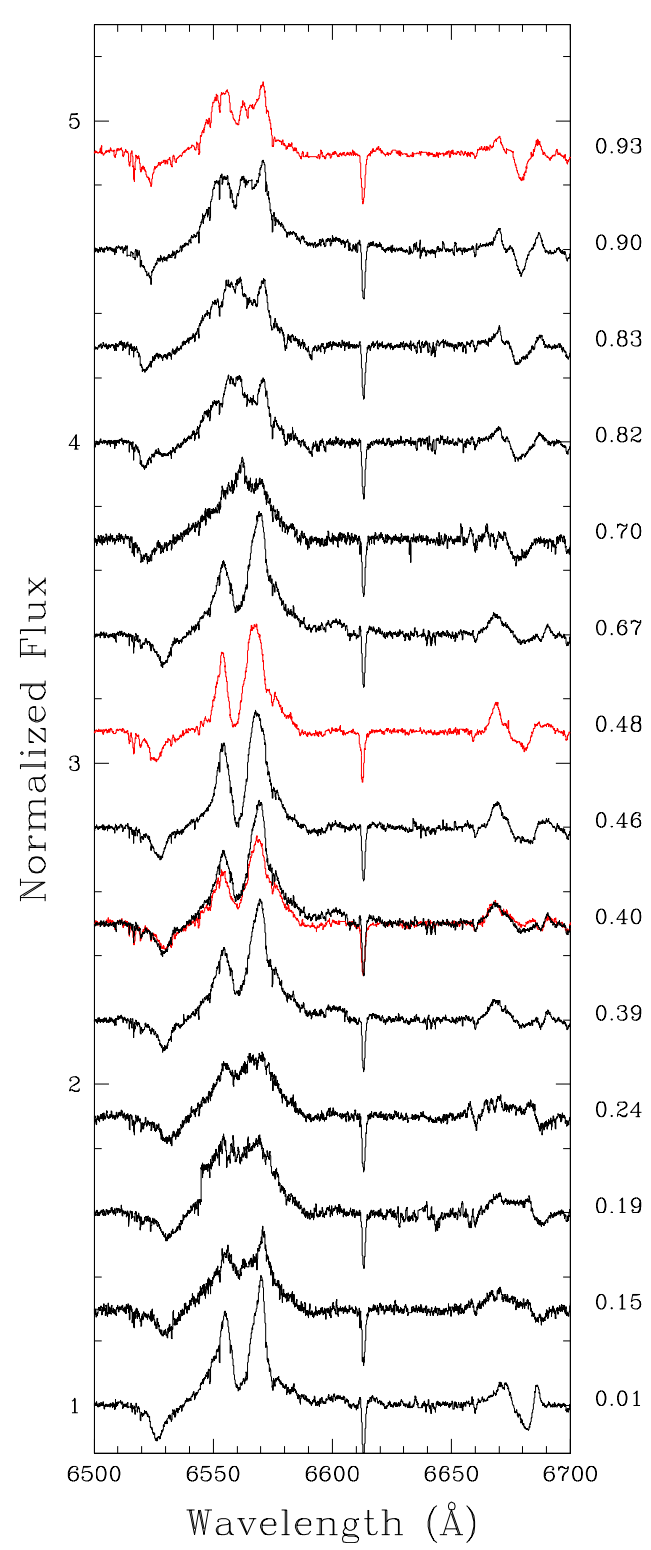

Wavelength $(\AA)$

Fig. 4. Line profile variations of some important emission lines in the optical spectrum of LSS 3074 . N III $\lambda \lambda 4634-4641$ and He II $\lambda 4686$ are shown in the left panel; He I $\lambda 5876$ is represented in the central panel; and $\mathrm{H} \alpha$ is indicated in the right panel. The orbital phases computed according to the ephemerides in Table 4 are given on the right of each panel. The red spectra correspond to the EMMI observations. The vertical solid (resp. dotted) lines in the middle panel represent the position of the primary (resp. secondary) He I $\lambda 5876$ line for the given observation.

lines lead to the addition of an $\mathrm{f}$ qualifier (see Walborn et al. 2002; Sota et al. 2011; Maíz Apellániz et al. 2016, and references therein) for both components. The primary spectrum further displays emissions of Si IV $\lambda \lambda 4089,4116$. Previously these features were indicated by an $\mathrm{f}^{+}$qualifier, but it has been suggested that this notation is obsolete (Sota et al. 2011).

However, the Conti criterion for the luminosity classes is formulated for spectral types from $\mathrm{O} 7$ to 09.7 and its application to the spectrum of the primary star is thus an approximation. Moreover, Walborn et al. (2002) argued that it would be preferable to use the $\mathrm{N}$ IV/N III emission line ratio for the spectral classification of the earliest O-type stars rather than the ratio of the He I/He II absorption lines. As shown in Fig. 3, the N III $\lambda \lambda$ 4634, 4641, and the very weak N IV $\lambda 4058$ emission line (when present) clearly move along with the primary star. The same holds true for the weak, but definite $\mathrm{N} v \lambda \lambda 4604$ and 4620 absorption lines. As indicated in Sect. 3, the presence of these $\mathrm{N} \mathrm{V}$ absorption lines points towards a rather hot star with an O3 to O4 spectral type (Walborn et al. 2002; Sota et al. 2011), which is at odds with our above classification based on the helium lines. We have thus compared our disentangled primary spectrum with the spectral atlas of Sota et al. (2011). The strong He II $\lambda 4686$ emission clearly confirms a supergiant luminosity class. The simultaneous presence of strong N III, very weak N IV, and definite $\mathrm{N} v$ lines in the spectrum of the primary is clearly a challenge both for spectral classification and spectral modelling (see Sect. 8.4). The best match, although certainly not perfect, is found with the spectra of HD 14947 (O4.5 If) and, to a lesser extent, HD 15570 (O4 If). For a highly deformed star, such as the primary of LSS 3074, gravity darkening leads to a non-uniform surface temperature. Hence, the discrepancy between the O5.5 If spectral type inferred from the relative strengths of the He I and He II lines and the O4-4.5 If type derived from the nitrogen spectrum could simply reflect the fact that these lines form over different parts of the stellar surface. We come back to this point in Sect. 8.4. For the secondary star, comparison with the atlas 

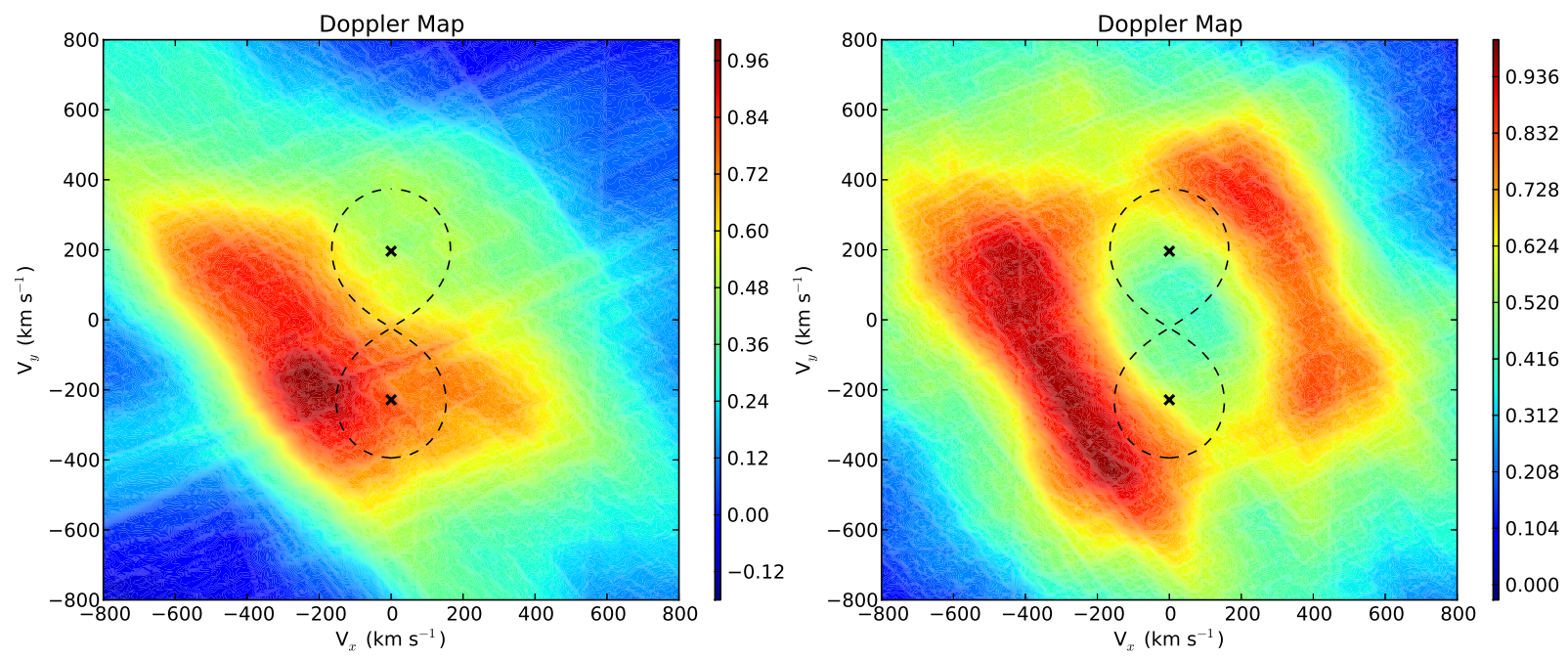

Fig. 5. Doppler maps of the He II $\lambda 4686$ (left) and H $\alpha$ (right) emission lines in the spectrum of LSS 3074 . The crosses correspond to the radial velocity amplitudes of the centre of mass of the primary (negative $v_{y}$ ) and secondary (positive $v_{y}$ ), whereas the dashed lines indicate the Roche lobe in velocity space for a mass ratio $m_{1} / m_{2}=0.86$. The colour scale indicates the level of the line emissivity relative to its maximum value.

of Sota el al. (2011) yields a spectral classification O6.5-7 If, in agreement with the results from the relative strengths of the helium lines.

Finally, since our classification is based on the disentangled spectra, it is less sensitive to a possible phase dependence of the line strengths and should thus be more robust than a classification based only on spectra collected near quadrature phases.

\subsection{Brightness ratio}

The spectral disentangling yields the strength of the lines in the primary and secondary stars relative to the combined continuum. But as we mentioned earlier, it does not allow us to establish the relative strengths of the continua. We therefore needed to first establish the brightness ratio of the stars to further analyse the reconstructed spectra.

To estimate the optical brightness ratio of the components of LSS 3074, we measured the equivalent widths, referring to the combined continuum of the two stars, of a number of spectral lines on the reconstructed primary and secondary spectra. The results are listed in Table 6 along with the mean equivalent widths of the same lines in synthetic spectra of stars of the same spectral type, computed with the non-LTE model atmosphere code CMFGEN (Hillier \& Miller 1998), which we describe in Sect. 8.2. For comparison, we also list the mean equivalent widths of the same lines in spectra of stars of similar spectral type as measured by Conti $(1973,1974)$ and Conti \& Alschuler (1971). from

The brightness ratio of the two stars can then be evaluated

$\frac{l_{1}}{l_{2}}=\left(\frac{E W_{1}}{E W_{2}}\right)_{\text {obs }}\left(\frac{E W_{O 7}}{E W_{O 5.5}}\right)_{\text {mean }}$.

By combining our measurements with those from synthetic spectra, we derive an optical brightness ratio of $2.50 \pm 0.43$. As a consistency check, we can also determine the brightness ratio through a comparison with the measurements made by Conti, and we obtain $2.61 \pm 0.41$.

The disentangled continuum normalized primary and secondary optical spectra are shown in Fig. 8.

\section{Light curve}

To improve our understanding of the system LSS 3074, and in particular its geometry, we performed an analysis of the photometric light curves by modelling the system using the eclipsing binary star simulator NIGHTFALL ${ }^{2}$, developed by Wichmann, Kuster and Risse. We worked in an iterative way. First of all, we fixed the mass ratio and orbital period to the values obtained from the orbital solution (see Table 5) and the effective temperatures to typical values for stars of similar spectral types (Martins et al. 2005). This permitted us to obtain a first estimation of the photometric solution and of the stellar radii and masses. Based on these approximated parameters, together with the effective temperatures, we calculated the associated surface gravities and stellar luminosities. This first approximation of the stellar fundamental parameters was then used as starting point input in our study of the atmosphere modelling procedure (see Sects. 8.2 and 8.3). This procedure then permitted us to accurately determine the effective temperatures of the stars, giving us a new input for the light curve study with NIGHTFALL.

Inspection of the light curve reveals a lack of a plateau between the minima, indicating important contributions of ellipsoidal variations to the photometric variability. Actually, the shape of the light curve suggests that almost the entire photometric variations could stem from such ellipsoidal variations, thereby implying that at least one of the stars must fill or overflow its Roche lobe.

The only evidence for the existence of genuine eclipses comes from the fact that the secondary minimum seems about 0.02 mag deeper than the primary minimum. The primary minimum corresponds to the occultation of the primary star by the secondary. What is surprising here is that the deeper minimum actually corresponds to the spectroscopically hotter star being in front. Given the level of dispersion in the light curve, it is quite possible that the small difference in depth of the minima is not significant and could arise, for example, from intrinsic variations of the stars. An alternative explanation for this situation could be an atmospheric eclipse produced by the wind of the primary

2 For more details, see the NIGHTFALL user manual by Wichmann (1998) available at the URL: http://www .hs . uni-hamburg .de/DE/ Ins/Per/Wichmann/Nightfall.html 
Table 6. Brightness ratio determination from the dilution of prominent lines.

\begin{tabular}{lccccccc}
\hline \hline Line & \multicolumn{7}{c}{ Equivalent width $(\AA)$} \\
\hline
\end{tabular}

Notes. The measured EWs are compared with values for the same lines in synthetic spectra of the same spectral type and in the compilation of measurements from the literature. The last column yields the brightness ratio for each line considered, using the synthetic spectra EWs.

star. Antokhina et al. (2013) studied the impact of free electron scattering in the wind of one star on the light curves of close eclipsing binaries. For a contact binary made of otherwise equal stars, these authors show that the presence of a stellar wind with $\dot{M}=10^{-5} M_{\odot} \mathrm{yr}^{-1}$ around one component deepens the eclipse corresponding to the star with the wind passing in front of its companion by about $0.08 \mathrm{mag}$. At the same time, the depth of the other eclipse is slightly reduced. In the case of LSS 3074, the fact that most emission lines closely follow the motion of the primary star suggests that this star has the strongest wind, thus lending support to this explanation. However, as pointed out by Antokhina et al. (2013), a quantitative assessment of the impact on the light curve requires some independent determination of the wind parameters (mass-loss rate, asymptotic velocity and exponent of velocity law). In our case, this is difficult to achieve (see Sect. 8). This is because of the lack of UV spectroscopy and the complexity of the line emission regions. Indeed, as revealed by our tomographic analysis, at least parts of the $\mathrm{H} \alpha$ and He II $\lambda 4686$ emissions arise from an interaction zone and these lines hence do not necessarily reflect the genuine properties of the winds. Yet, our best-fit model atmosphere parameters (see the forthcoming Table 10) yield upper limits on the wind density that could be consistent with the observed difference in eclipse depth. However, these best-fit parameters also suggest that both binary components lose material at a similar rate. Hence, one would expect both eclipses to be affected by stellar wind absorption. We thus conclude that a better knowledge of the wind parameters of LSS 3074 is required before we can attempt a model of the light curve accounting for the effects of the stellar winds. Whatever the origin of the slight difference in depth of the minima, we need to keep in mind that this situation affects the quality of the fits of the light curve.

To explain the shape of the secondary minimum by the sole effect of ellipsoidal variations, we at least need to assume that either both components of the binary system fill up (contactcontact) or even overflow their Roche lobes (overcontact), or that one of the components must be much smaller and much fainter than the other star that fills up its Roche lobe. In the former case, one expects both stars to be of very comparable size and, given their effective temperatures, also of rather comparable brightness. To explain the depth of almost 0.2 mag by the sole effect of ellipsoidal variations is actually not possible here without requiring the presence of true eclipses. In this context, the necessity to invoke a contact-contact or an overcontact configuration remains valid, along with the conclusion that sizes and brightness of both stars should be comparable.

This is precisely the outcome of our fits with the NIGHTFALL code. The best-fit quality $\left(\chi_{v}^{2}=4.4\right)$ is achieved for
Table 7. Photometric solution for LSS3074.

\begin{tabular}{ccc}
\hline \hline Parameters & Primary & Secondary \\
\hline$i\left(^{\circ}\right)$ & \multicolumn{2}{c}{$54.5 \pm 1.0$} \\
$q=m_{1} / m_{2}$ & \multicolumn{2}{c}{0.86 (fixed) } \\
Filling factor $^{a}$ & $1.008 \pm 0.010$ & $1.008 \pm 0.010$ \\
$T_{\text {eff }}(\mathrm{K})$ & 39900 (fixed) & 34100 (fixed) \\
$m\left(M_{\odot}\right)$ & $14.8 \pm 1.1$ & $17.2 \pm 1.4$ \\
$R_{\text {pole }}\left(R_{\odot}\right)$ & 7.8 & 8.4 \\
$\chi^{2}$ & \multicolumn{2}{c}{1820.7} \\
$N_{\text {d.o.f. }}$ & \multicolumn{2}{c}{415} \\
\hline
\end{tabular}

Notes. $N_{\text {d.o.f. }}$ is the number of degrees of freedom. ${ }^{(a)}$ The filling factor of a given binary component is defined here as the fraction of its polar radius over the polar radius of its Roche lobe.

overcontact configurations with a filling factor of $1.008(i \simeq$ $54.5^{\circ}$ ), where the corresponding $V$-band brightness ratio is predicted to be 1.09. The corresponding photometric solution is presented in Table 7 and the associated plot is shown in Fig. 6. Yet, our spectroscopic analysis suggests an optical brightness ratio (primary/secondary) near 2.50. This situation is thus clearly at odds with explaining the photometric light curve via a double contact or overcontact configuration. To achieve a brightness ratio of $2.50 \pm 0.43$, the ratio between the mean radii of the primary and secondary stars would have to be $1.15 \pm 0.10$. This translates into a ratio of the filling factors of $1.23 \pm 0.11$, which is only possible for detached or semi-detached configurations. As could be expected, the fact that the hotter star is in front during the deepest minimum leads to difficulties to fit this minimum.

Since the eclipses are only partial, one way to solve this issue would be to postulate the existence of a dark spot on the side of the secondary star facing the primary. Such a spot would then lead to a deeper minimum when the primary star is in front. From a purely numerical point of view this would improve the fit quality significantly with $\chi_{v}^{2}$ now approaching 2.9 . In addition, it would certainly help to bring the spectroscopic and photometric brightness ratios into better agreement. Yet, aside from the difficulty of explaining the physical origin of such a spot, there is another issue that concerns the fact that the spot size and its dim factor (i.e. the reduction in local temperature) are not fully independent and we lack any objective constraints on these parameters. Test calculations have shown that for some situations, the best-fit model would actually correspond to a spot that covers more than $75 \%$ of the surface of the secondary, which is clearly not physical.

As an alternative to the overcontact configuration, one might consider a scenario where the primary star overflows its Roche 


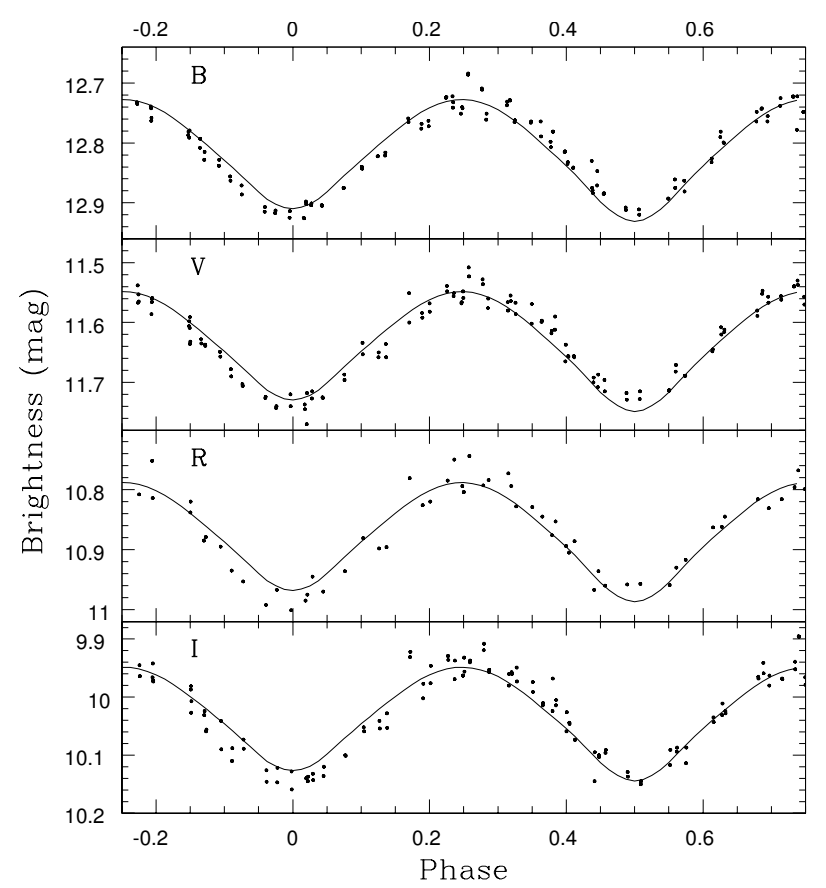

Fig. 6. Photometry of LSS 3074 . The observational data are presented as black dots, and the solid black line corresponds to the best-fit theoretical light curve fit with NIGHTFALL with the parameters presented in Table 7. The zero phase corresponds to the secondary eclipse, when the primary is occulting the secondary.

lobe and transfers matter to a geometrically thick accretion disk around the more massive secondary star. This kind of scenario has been proposed to explain the light curves of $\beta$ Lyrae (Wilson 1974), RY Sct (Antokhina \& Kumsiashvili 1999), and V455 Cyg (Djurašević et al. 2012), among others. On the positive side of such a scenario, it might help reconcile the photometric and spectroscopic brightness ratios. On the negative side, we have the lack of a clear signature of an accretion disk in the Doppler maps of the $\mathrm{H} \alpha$ line and the fact that LSS 3074 is a very compact system, leaving very little room for the formation of a disk, unless the secondary star is very small. To test this scenario, we used the disk option in the NIGHTFALL code. In addition to the effective temperatures that we fixed at the values obtained from our model atmosphere fits (see Sect. 8.3), we set the Roche lobe filling factor of the primary star to unity and that of the secondary star to 0.75 . This choice results in a brightness ratio that is consistent with our spectroscopic value. We tested various options for the disk available in the NIGHTFALL code (simple disk with uniform temperature, isothermal disk and reprocessing disk), which correspond to different prescriptions of the variations of the disk height and temperature with radius. Since the disk in our models resembles more an annulus than a genuine disk, these three options actually yield nearly identical results. These models fail to reproduce the width of the secondary minimum. This failure increases the $\chi^{2}$ of the fits. Still, we managed to find fits with $\chi_{v}^{2} \sim 4.6$, but this was only possible for a disk temperature exceeding the temperatures of both stars, which is most probably not physical ${ }^{3}$. Better quality fits can be obtained if the Roche lobe filling factor of the secondary is allowed to drop below 0.75 : we obtained $\chi_{v}^{2}=3.9$ for a secondary filling factor of 0.47 (which would correspond to a secondary radius of

\footnotetext{
3 Raising the disk temperature allows the model to better reproduce the depth of the primary minimum, hence partially compensating for the increase of $\chi^{2}$ due to the poor fit of the secondary minimum.
}

Table 8. Fit of the light curves of LSS 3074 by a sine function.

\begin{tabular}{rcccc}
\hline \hline & \multicolumn{4}{c}{ Filter } \\
\hline$P=1.0926 \mathrm{~d}$ & $B$ & & $R$ & $I$ \\
$a(P)$ & 0.0901 & 0.0910 & 0.0964 & 0.0980 \\
$\sigma_{a}$ & 0.0022 & 0.0022 & 0.0039 & 0.0027 \\
$\chi_{v}^{2}$ & 5.96 & 2.92 & 2.65 & 2.66 \\
$\sigma_{\text {fit }}$ & 0.0171 & 0.0171 & 0.0212 & 0.0212 \\
\hline$P=2.1852 \mathrm{~d}$ & & & & \\
$a_{1}(P)$ & 0.0118 & 0.0125 & 0.0107 & 0.0125 \\
$\sigma_{a_{1}}$ & 0.0020 & 0.0020 & 0.0038 & 0.0020 \\
$a_{2}(P / 2)$ & 0.0896 & 0.0905 & 0.0958 & 0.0905 \\
$\sigma_{a_{2}}$ & 0.0019 & 0.0019 & 0.0037 & 0.0019 \\
$\chi_{v}^{2}$ & 4.60 & 2.20 & 2.38 & 1.30 \\
$\sigma_{\text {fit }}$ & 0.0150 & 0.0148 & 0.0200 & 0.0148 \\
\hline$N$ & 120 & 122 & 58 & 118 \\
$\sigma$ & 0.007 & 0.010 & 0.013 & 0.013 \\
\hline
\end{tabular}

Notes. $P$ is the adopted period, $a_{i}$ the semi-amplitudes, $\sigma_{a_{i}}$ the $1 \sigma$ error on semi-amplitude, $\chi_{v}^{2}$ the minimum reduced $\chi^{2}, \sigma_{\text {fit }}$ the rms of the residuals, $N$ the number of points and $\sigma$ the expected error.

only $3.9 R_{\odot}$ ) and a disk temperature of $51300 \mathrm{~K}$. Both parameters (secondary radius and disk temperature) are most probably not physical. We thus conclude that a disk model cannot solve the issues raised above any better than the pure Roche potential model.

From a purely phenomenological point of view, we can expand the light curve into a sum of sine functions. We tried a period of $2.1852 / 2=1.0926$ days and a mere sine and a period of 2.1852 days and the fundamental sine plus its harmonic. The results are given in Table 8 . The photometric observations exhibit a variation with a semi-amplitude of 0.09 mag in all filters. The semi-amplitude associated with the modulation on the orbital period is $0.01 \mathrm{mag}$ reflecting the above-mentioned difference between the depths of the two minima. The corresponding $\chi_{v}^{2}$ are lower than for any other fit we tried but still not satisfactory. Including a larger number of harmonics in our expansion does not improve the situation. However, in terms of standard binary models, this fit is unphysical since a modulation with a semi-amplitude as large as 0.09 mag cannot be explained by ellipsoidal variations for such models. The fit of the $B$ light curve corresponds to a larger $\chi_{v}^{2}$; the corresponding $\chi_{v}^{2}$ would be similar to those found for the data from the other filters provided that the photometric errors used to normalize the $\chi_{v}^{2}$ were $0.010 \mathrm{mag}$ instead of $0.007 \mathrm{mag}$.

The $\chi_{v}^{2}$ of Table 8 are too large and this anomaly could have several origins. One possibility is that the above-mentioned photometric errors deduced from the comparison stars might not apply to the case of LSS 3074. This could be due to an unfortunate location on the CCDs or for example to strange behaviour due to peculiar stellar colours. This interpretation is however not very likely. Alternatively, the fitted mathematical model might not be correct. Since any periodic function can be expanded into a Fourier series and we found that our sine fits do not require additional terms, the additional, non-periodic component of the model behaves similarly to an observational noise although it must have a different origin. A possible explanation could be an intrinsic variability of the star at high frequencies since no low-frequency variations were previously detected beyond the orbital one. Of course, this additional component has an upper limit. Since the sine fits are our best models, we can consider 
estimating this upper limit from the corresponding residuals. In the $V$ filter for instance, we would need an additional variability compared to the expected $\sigma=0.010 \mathrm{mag}$ photometric error that quadratically adds to it to reach $\sigma_{\text {fit }}=0.0148 \mathrm{mag}$ for the two periods fit or $\sigma_{\text {fit }}=0.0171 \mathrm{mag}$ for the single period fit. Considering that the difference in depth between the two minima is not well established, we can adopt the latter and the relevant value would thus be $\sigma_{\text {fit }}=0.0171 \mathrm{mag}$.

The transition from $\sigma=0.010$ to $0.0171 \mathrm{mag}$ translates into a change from $\sigma=0.009$ to $\sigma^{\text {corr }}=0.0185 \mathrm{mag}$ for the global fit of the data from all four filters. The substitution by these newly estimated errors alleviates the rejection of the NIGHTFALL model, since it reduces the $\chi^{2}$. However, this is purely artificial. In addition, the $\chi_{v}{ }^{2}$ of 4.4 could still be due to a lack of ability of the NIGHTFALL model to fit the light curve, even if it were noiseless. However, under the hypothesis of the fit of the NIGHTFALL model, the errors on the parameters must be modified to remain coherent. Even though this additional dispersion does not stem from the same origin as the genuine photometric errors, impact of this dispersion on the derived parameters is similar to that of photometric errors.

To obtain the $1 \sigma$ error on the parameters of the NIGHTFALL models, we have to consider the dispersion at $\chi_{\min }^{2}+\Delta \chi_{1 \sigma}^{2}$ where $\Delta \chi_{1 \sigma}^{2}=2.3$ for the $1 \sigma$ confidence interval of the simultaneous adjustment of two free parameters $\left(i\right.$ and $f i l l_{\mathrm{p}}=f i l l_{\mathrm{s}}$ as is the case when we fit the light curve assuming an overcontact configuration with NIGHTFALL). From the values of the $\chi^{2}$ of our best-fit NIGHTFALL model, the $1 \sigma$ error bars on the orbital inclination and the filling factors would be unrealistically small (e.g. about $0.1^{\circ}$ for the error on $i$ ). However, as pointed out above, the dispersion of the photometric data about the best-fit synthetic light curve exceeds the value of our estimated photometric errors, suggesting that there could be an intrinsic photometric variability in addition to the orbital modulation.

Admitting the impact of the additional dispersion, we have to correct the $\Delta \chi_{1 \sigma}^{2}=2.3$ to take this effect into account. Therefore, we have to adopt a corrected $\Delta \chi_{1 \sigma \text {, corr }}^{2}=\Delta \chi_{1 \sigma}^{2} \times\left(\frac{\sigma^{\text {corr }}}{\sigma}\right)^{2}=8.9$. We have then estimated the errors on the NIGHTFALL model parameters by accordingly adopting this new corrected value. In this way, rounding the values uppards, we estimate errors of $1.0^{\circ}$ and of 0.01 for the inclination and the filling factors of both stars, respectively.

\section{Spectral analysis}

\subsection{Rotational velocities and macroturbulence}

In order to determine the projected rotational velocities $(v \sin i)$ of the stars of the system, we applied a Fourier transform method (Simón-Díaz \& Herrero 2007; Gray 2008). For the primary star, we used the profiles of the He I $\lambda 4471$, He II $\lambda \lambda 4200,4542$, 6118 , and N III $\lambda 6075$ lines of the reconstructed spectrum. We selected these lines as they are rather well isolated in the spectra and should thus be free of blends. Unfortunately, we could not use the same lines for the secondary star because they are particularly deformed. Indeed, the red wing of these lines is steeper than the blue wing. We then used the He II $\lambda 5412$ and Si IV $\lambda 4089$ lines to determine the projected rotational velocity of the secondary star. The results are presented in Table 9 . The mean $v \sin i$ of the primary star is $(110 \pm 13) \mathrm{km} \mathrm{s}^{-1}$, while that of the secondary is $(127 \pm 6) \mathrm{km} \mathrm{s}^{-1}$. Owing to the small number of lines usable to study the rotation of the secondary star, we could not use a classical standard deviation method to determine the
Table 9. Projected rotational velocities $\left(v \sin i\right.$ expressed in $\left.\mathrm{km} \mathrm{s}^{-1}\right)$ of the components of LSS 3074.

\begin{tabular}{ccc}
\hline \hline Line & Primary & Secondary \\
\hline Si IV $\lambda 4089$ & - & 126 \\
He II $\lambda 4200$ & 115 & - \\
He I $\lambda 4471$ & 97 & - \\
He II $\lambda 4542$ & 119 & - \\
He II $\lambda 5412$ & - & 128 \\
N III $\lambda 6075$ & 92 & - \\
He II $\lambda 6118$ & 125 & - \\
\hline Mean value & $110 \pm 13$ & $127 \pm 6$ \\
\hline
\end{tabular}

associated error bars. We thus studied the Fourier transforms for a sample of rotational velocities to estimate these errors. From these values of $v \sin i$ and considering the inclination of the system from Sect. 7, we found that the stars are in synchronous rotation with a rotational period very close to the orbital period of the system.

Macroturbulence is defined as a non-thermal motion in the stellar atmosphere with turbulent cells larger than the mean free-path of the photons. The main effect of macroturbulence is an additional broadening of the spectral lines. An approximation of the macroturbulence velocities was obtained by applying the radial-tangential anisotropic macroturbulent broadening formulation of Gray (2008) on the spectra, after the inclusion of rotational velocity broadening. For this purpose, we used the auxiliary program MACTURB of the stellar spectral synthesis program SPECTRUM v2.76 developed by Gray (2010). We applied this technique to the lines $\operatorname{He} I \lambda \lambda$ 4026, 4471, and 5016, and He II $\lambda 4542$ and obtained macroturbulence velocities of 20 and $50 \mathrm{~km} \mathrm{~s}^{-1}$, for the primary and secondary stars, respectively.

Both rotational and macroturbulence velocities were applied on the synthetic spectra (see Sect. 8.2) before comparing the latter with the disentangled spectra.

\subsection{CMFGEN code}

In order to determine the fundamental properties of both components of LSS 3074, we used the non-LTE model atmosphere code CMFGEN (Hillier \& Miller 1998). This code solves the equations of radiative transfer and statistical equilibrium in the co-moving frame, is designed to work for both plane-parallel and spherical geometries, and can be used to model Wolf-Rayet stars, O stars, luminous blue variables and supernovae. A super-level approach is adopted for the resolution of the equations of statistical equilibrium. The CMFGEN code also further accounts for line blanketing and its impact on the energy distribution. The hydrodynamical structure of the stellar atmosphere is directly specified as an input to the code, and a $\beta$ law is used to describe the velocity law within the stellar winds. We included the following chemical elements and their ions in our calculations: $\mathrm{H}$, $\mathrm{He}, \mathrm{C}, \mathrm{N}, \mathrm{O}, \mathrm{Ne}, \mathrm{Mg}, \mathrm{Al}, \mathrm{Si}, \mathrm{S}, \mathrm{Ca}, \mathrm{Fe}$, and $\mathrm{Ni}$. The solution of the equations of statistical equilibrium is used to compute a new photospheric structure, which is then connected to the same $\beta$ wind velocity law. The radiative transfer equations were solved based on the structure of the atmosphere with a microturbulent velocity varying linearly with wind velocity from $20 \mathrm{~km} \mathrm{~s}^{-1}$ in the photosphere to $0.1 \times v_{\infty}$ at the outer boundary, and generated synthetic spectra were compared to the reconstructed spectra of the primary and secondary stars. 
As a first approximation, the mass-loss rates and $\beta$ parameters were taken from Muijres et al. (2012) for the spectral types of both stars, whilst the wind terminal velocity was assumed equal to the mean value for stars of the same spectral type (Prinja et al. 1990). We then estimated the surface gravities, stellar masses, radii, and luminosities from our study of the photometric curves. The relevant parameters were then adjusted via an iterative process, as each adjustment of a given parameter leads to some modifications in the value of other parameters. This process and the results we obtained are presented in Sects. 8.3 and 8.4, respectively. As explained in Sect. 6, this iterative process was then coupled with a second iterative process, through the study of the photometric light curve.

\subsection{Method}

The first step is to adjust the effective temperature in the models of the stars. To do so, we adjusted the relative strengths of the He I $\lambda 4471$ and He II $\lambda 4542$ lines (Martins 2011). Final values are 39900 and $34100 \mathrm{~K}$ for the primary and secondary stars, respectively. Once the effective temperatures were determined, we used them, together with the stellar radii obtained in our study of the photometric curves, to constrain the luminosities of the stars. We therefore obtained luminosities of $1.38 \times 10^{5} L_{\odot}$ and $8.49 \times 10^{4} L_{\odot}$ for the primary and secondary stars.

Subsequently, the binarity of the studied system causes some problems. Indeed, the next step is to adjust the surface gravities in the models, but as we mentioned in Sect. 6.1, the wings of the Balmer lines are too broad to be properly recovered by the spectral disentangling. To circumvent this problem, we recombined the models of the primary and secondary star spectra for several phases and compared the resulting binary spectra directly to the observations. This permitted us to better adjust the gravities, but with larger uncertainties than in the case of a single star.

A second problem arises with the adjustment of the wind parameters. Indeed, the He II $\lambda 4686$ and Balmer lines may be polluted by some emission from the wind-wind interaction zone. Therefore, the uncertainties on the terminal velocity, the $\beta$ of the velocity law, the clumping factor, the clumping velocity factor, and the mass-loss rate are quite high. Indeed, considering the possible existence of such additional emission polluting these diagnostic lines, the adjustment of the models onto the reconstructed spectra may lead to an underestimate of the terminal velocity, and an overestimate of the $\beta$ of the velocity law, clumping factor, clumping velocity factor, and mass-loss rate. Therefore, the obtained values can only be considered as lower and upper limits of the real properties of the stellar winds. However, we still adjusted them as well as possible: we obtained for the primary and secondary stars values of 2615 and $3055 \mathrm{~km} \mathrm{~s}^{-1}$ for $v_{\infty}, 1.30$ and 1.40 for $\beta$ and $3.00 \times 10^{-6}$ and $3.51 \times 10^{-6} M_{\odot} \mathrm{yr}^{-1}$ for the mass-loss rate, based on the strength of $\mathrm{H} \alpha$, the width of the He II $\lambda 4686$ and $\mathrm{H} \alpha$ lines, and both the strengths of $\mathrm{H} \gamma$ and $\mathrm{H} \delta$, respectively. The clumping formalism used in the CMFGEN model is

$f(r)=f_{1}+\left(1-f_{1}\right) \mathrm{e}^{\left(-\frac{V(r)}{f_{2}}\right)}$

with a clumping filling factor $f_{1}$ of 0.7 , a clumping velocity factor $f_{2}$ of $200 \mathrm{kms}^{-1}$ and $V(r)$ the velocity of the wind for both primary and secondary stars, and we adjusted these two clumping factors through the strength and shape of $\mathrm{H} \alpha$ and $\mathrm{H} \beta$ lines.

Finally, once the fundamental properties of the stars had been established or fixed, we investigated the $\mathrm{CNO}$ abundances within their atmosphere through the strengths of associated lines. At that point, we encountered several problems. Indeed, there is no visible $\mathrm{O}$ line in both primary and secondary spectra, and the only present $\mathrm{C}$ lines are the C IV $\lambda \lambda 5801$ and 5812 lines, which cannot be considered in the determination of the surface $C$ abundance since they can exhibit complex profiles influenced by various phenomena among which is continuum fluorescence (Conti 1974; Bouret et al. 2012). We thus were only able to determine upper limits for $\mathrm{C}$ and $\mathrm{O}$ abundances. We used the N III $\lambda 4379$ and N III $\lambda \lambda 4511-15-18-24-30-35$ lines to adjust the $\mathrm{N}$ abundance for the primary star. The same lines were used for the secondary star except for the N III $\lambda 4530$ and N III $\lambda 4535$ lines. These $N$ abundance diagnostic lines are taken from Martins et al. (2015) study of a sample of 74 objects comprising all luminosity classes and spectral types from $\mathrm{O} 4$ to 09.7 . We selected, from their study, all the suggested lines that were significantly seen in our observations. We performed a normalized $\chi^{2}$ analysis to determine the best fit to these lines (Martins et al. 2015). The normalization consists in dividing the $\chi^{2}$ by its value at minimum, $\chi_{\min }^{2}$. As a $1 \sigma$ uncertainty on the abundances, we then considered abundances up to a $\chi^{2}$ of 2.0, i.e. an approximation for 1 over $\chi_{\min }^{2}$, as suggested by Martins et al. (2015).

\subsection{Results}

Figure 8 shows the best fit of the optical spectra of the primary and secondary stars obtained with CMFGEN, and we present in Table 10 the associated stellar parameters.

Figure 8 reveals that we encountered a lot of difficulty adjusting the spectra of the components of LSS 3074. First of all, it clearly shows that the noise is strongly enhanced in the reconstructed secondary spectrum, because of the high brightness ratio of the system. This peculiarity makes the adjustment of the secondary spectrum even more difficult. Second, if we can see that the He lines are generally well reproduced for both stars, some of these lines in the observed spectra, especially for the secondary star, feature some emission in their wings that is not reproduced by the models. We can also see that the Balmer and He II $\lambda 4686$ lines are not perfectly well recovered, probably owing to the complexity of the winds and the presence of a windwind interaction zone, as discussed in Sect. 8.3.

Concerning the $\mathrm{N}$ lines, most $\mathrm{N}$ III features are reasonably well reproduced by the models, but a few lines of other nitrogen ions are not, especially in the primary spectrum. Indeed, the model predicts a rather strong N IV $\lambda 4058$ emission, while the observations clearly show that this is not the case. Another remarkable issue is the presence in the observed primary spectra of the $\mathrm{NV} \lambda \lambda 4604,4620$ lines, which are not predicted by the model. These N V lines are seen in the spectra of the earliest $\mathrm{O}$ supergiants (starting from O4 If Walborn et al. 2002; Sota et al. 2011) and, in some cases, in the spectra of early $\mathrm{O}$ mainsequence stars (e.g. Mahy et al. 2012), and could suggest that our value of the primary effective temperature $(39900 \mathrm{~K})$ is too low. To test this hypothesis, we computed a set of CMFGEN models with temperatures ranging from 38000 to $44000 \mathrm{~K}$. As one can see in Fig. 7, a temperature of about $44000 \mathrm{~K}$ would be needed to reproduce the strength of the $\mathrm{N} \mathrm{V}$ lines. However, in this case, the model predicts N III emissions that are much weaker than observed as well as a huge N IV line that is clearly not observed. To reproduce the strength of the observed N III and N IV lines, a lower temperature of $38000 \mathrm{~K}$ seems more indicative. But this lower temperature is clearly not sufficient to produce the $\mathrm{NV}$ lines. The temperature that we determined in our CMFGEN fits is the temperature that properly reproduces 

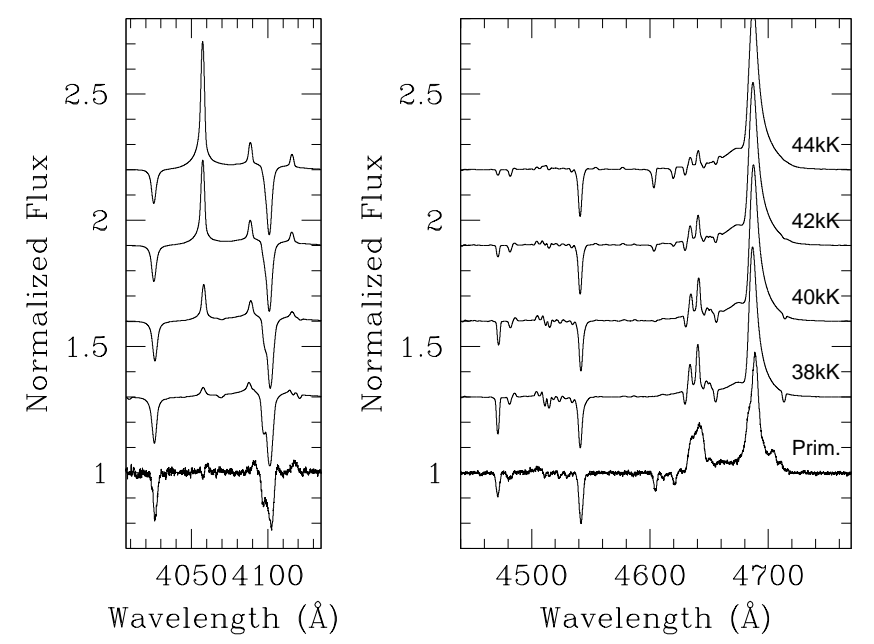

Fig. 7. Evolution of the strength of the N III $\lambda \lambda 4634-41$, N IV $\lambda 4058$, and $\mathrm{N} v \lambda \lambda 4604,4620$ lines in synthetic CMFGEN spectra as a function of effective temperature. The primary spectrum of LSS 3074 obtained via disentangling is shown at the bottom for comparison.

the strength of the helium lines and represents a compromise between the contradicting trends of the nitrogen lines.

Our difficulty in reproducing simultaneously the lines of $\mathrm{N}$ III, N IV, and N V in the spectra of $\mathrm{O}$ supergiants is actually not new. Crowther et al. (2002) and Rauw et al. (2002) encountered similar problems in their attempts to fit the spectra of the O4 $\mathrm{Iaf}^{+}$star HDE 269698 and of the O7 + O4 If/WN8ha binary HDE 228 766, respectively. More recently, Bouret et al. (2012) applied the CMFGEN code to a sample of early O supergiants. These authors also failed to reproduce consistently the lines of the three nitrogen ions in the spectra of the three O4 If supergiants HD 15 570, HD 16691, and HD 190 429A for which they derived effective temperatures of 38000,41000 , and $39000 \mathrm{~K}$, respectively. We note that these effective temperatures are very close to our best-fit value for the primary of LSS 3074.

The origin of these difficulties is currently unclear. One possibility could be that the models do not account (correctly) for the Auger effect. X-ray emission arising in the wind and back illuminating the stellar photosphere could lead to an overionization of nitrogen and favour the formation of the $\mathrm{N} v$ lines. In the specific case of the primary of LSS 3074, the simultaneous presence of the three ionization stages of nitrogen could also be from a non-uniform surface temperature as a result of gravity darkening. Applying the von Zeipel theorem to the primary, we find that the effective temperature near the poles should be about $15 \%$ higher than near the equator. The observed spectrum of the primary results from the combination of the spectra emitted by the various regions, which have their own temperatures (Palate \& Rauw 2012; Palate et al. 2013). Therefore, gravity darkening could indeed result in a spectrum with an unusual combination of spectral lines.

However, we can stress three very interesting results from our spectral analysis. First of all, as we can see in Table 10, we confirm the very low masses and radii predicted by Morrell \& Niemela (1990), which is unusual for stars with such spectral types. We can also see that the system seems to display strong winds, even though these winds are not perfectly constrained. Finally, we found a strong overabundance in $\mathrm{N}$ and a strong depletion in $\mathrm{C}$ and $\mathrm{O}$ in both stars of the system, together with a strong He enrichment of the primary atmosphere.
Table 10. Best-fit CMFGEN model parameters of the primary and secondary stars.

\begin{tabular}{ccc}
\hline \hline & Prim. & Sec. \\
\hline$R\left(R_{\odot}\right)$ & $7.5 \pm 0.6$ & $8.2 \pm 0.7$ \\
$M\left(M_{\odot}\right)$ & $14.6 \pm 2.1$ & $17.2 \pm 3.0$ \\
$T_{\text {eff }}\left(10^{4} \mathrm{~K}\right)$ & $3.99 \pm 0.15$ & $3.41 \pm 0.15$ \\
$\log (g)(\mathrm{cgs})$ & $3.82 \pm 0.20$ & $3.83 \pm 0.20$ \\
$\log \left(\frac{L}{L_{\odot}}\right)$ & $5.14 \pm 0.07$ & $4.93 \pm 0.08$ \\
$\beta$ & $\leq 1.30$ & $\leq 1.40$ \\
$v_{\infty}\left(\mathrm{km} \mathrm{s}^{-1}\right)$ & $\geq 2615$ & $\geq 3055$ \\
$\dot{M} / \sqrt{f_{1}}\left(M_{\odot} \mathrm{yr}^{-1}\right)$ & $\leq 3.00 \times 10^{-6}$ & $\leq 3.51 \times 10^{-6}$ \\
\hline
\end{tabular}

Notes. The quoted errors correspond to $1 \sigma$ uncertainties.

Table 11. Chemical abundances of the components of LSS 3074.

\begin{tabular}{cccc}
\hline \hline & Primary & Secondary & Sun \\
\hline $\mathrm{He} / \mathrm{H}$ & 0.25 & 0.09 & 0.089 \\
$\mathrm{C} / \mathrm{H}$ & $\leq 6.45 \times 10^{-5}$ & $\leq 2.05 \times 10^{-5}$ & $2.69 \times 10^{-4}$ \\
$\mathrm{~N} / \mathrm{H}$ & $5.65_{-3.02}^{+4.07} \times 10^{-4}$ & $3.69_{-0.91}^{+0.83} \times 10^{-4}$ & $6.76 \times 10^{-5}$ \\
$\mathrm{O} / \mathrm{H}$ & $\leq 2.67 \times 10^{-5}$ & $\leq 1.00 \times 10^{-5}$ & $4.90 \times 10^{-4}$ \\
\hline
\end{tabular}

Notes. Abundances are given by number as obtained with CMFGEN. The solar abundances (Asplund et al. 2009) are quoted in the last column. The $1 \sigma$ uncertainty was set to abundances corresponding to a normalized $\chi^{2}$ of 2.0 .

\section{Discussion and conclusions}

Our analysis of the light curve of LSS 3074 indicates that this system is a candidate for an overcontact binary. Massive overcontact binaries are important objects as they could be progenitors of merger events or of binary black hole systems (Lorenzo et al. 2014; Almeida et al. 2015). Yet, only a handful of such systems are known and sometimes the exact configuration (double contact versus semi-detached) is controversial (e.g. Yaşarsoy \& Yakut 2013; and Zhao et al. 2014, respectively). Among the best-studied systems hosting O-type stars are HD 100213 (=TU Mus, Linder et al. 2007), VFTS 352 (Almeida et al. 2015), and MY Cam (=BD $+56^{\circ} 864$, Lorenzo et al. 2014). Lorenzo et al. (2014) and Almeida et al. (2015) note that the components of MY Cam and VFTS 352 appear hotter than one would expect from their masses. This discrepancy also exists for LSS 3074 and is even worse than in the other systems. Lorenzo et al. (2014) interpret this result as a consequence of the highly elongated shape of the stars. In the case of VFTS 352, Almeida et al. (2015) further report an enhanced He abundance. These authors interpret this situation as the result of enhanced mixing in a tidally locked binary (de Mink et al. 2009) and suggest that the system is currently in a long-lived overcontact phase of case A mass transfer. Again LSS 3074 also shows abundance anomalies indicating that both stars are evolved (enhanced $N$ abundance) and the primary is the more evolved star (enhanced He abundance).

\subsection{Evolutionary status}

We first remark that we did not consider the possibility of quasichemically homogeneous evolution as described in Heger et al. (2000), because the observed rotational velocities of both components are too small. 

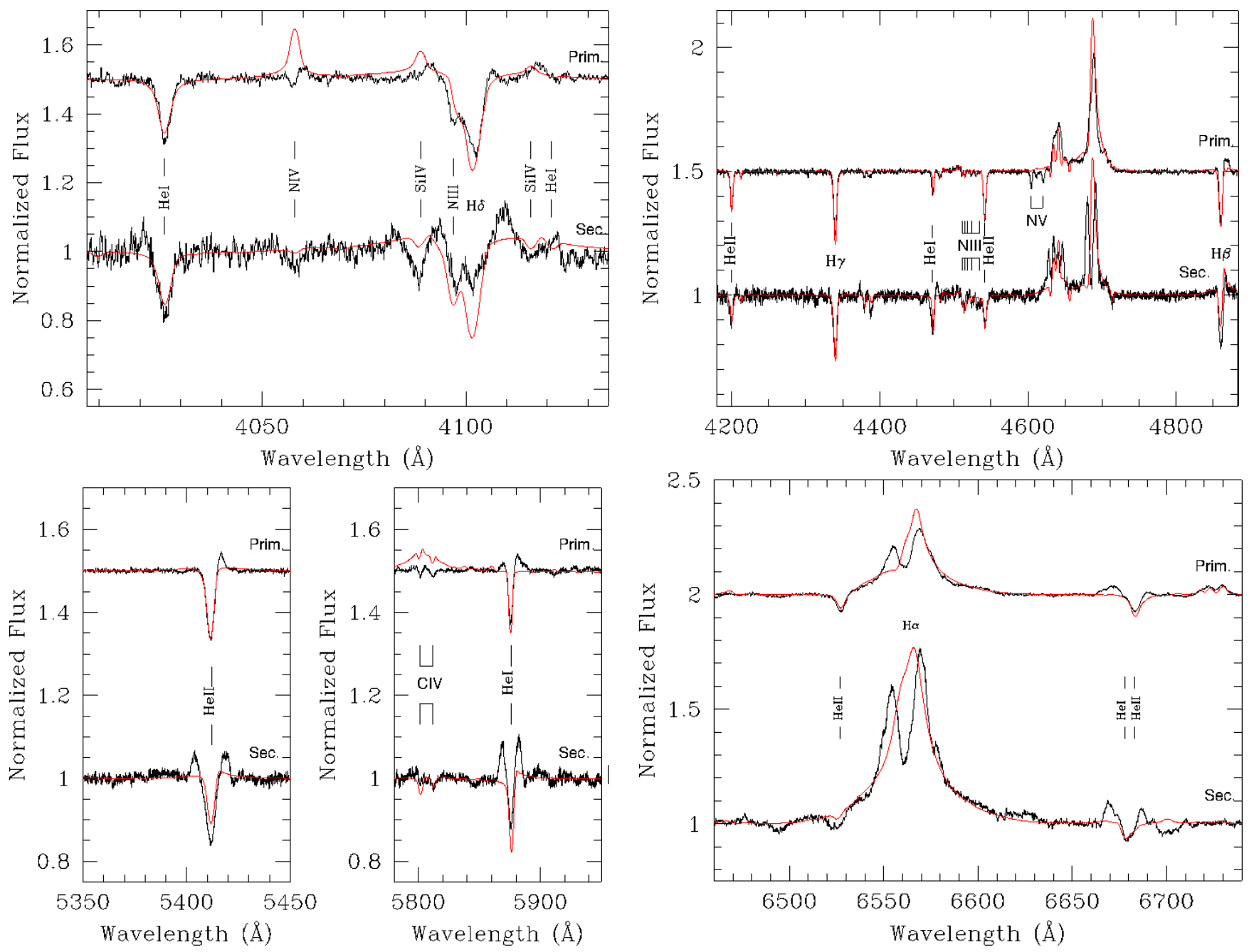

Fig. 8. Part of the normalized separated spectra of the primary (top, shifted upwards by 0.5 continuum units in the first three panels, and by 1.0 continuum units for the last panel) and secondary stars (bottom), along with the best-fit CMFGEN model spectra (red).

The altered CNO abundances in the atmosphere of the primary, the luminosity of primary, and the increased helium abundance are strong arguments that the star is in the slow phase of case B RLOF. If the star were post-RLOF, accounting for the present mass, its luminosity would have to be $\log \left(\frac{L}{L}\right)=5.5$ rather than the observed 5.14 (e.g. Vanbeveren et al. 1998, formula 5.4). Moreover, the observed CNO abundances of the secondary seem to indicate that at least some mass lost by the primary was accreted by the secondary. The normal (solar) helium abundance, on the other hand, lead us to conclude that not all the helium enhanced layers lost by the primary were accreted by the secondary. Accounting for these facts a theoretical study can be undertaken to attempt to identify its possible progenitors. For this purpose, the Brussels binary population synthesis code is used. This code uses as input thousands of detailed evolutionary calculations performed with the Brussels binary evolution code. Both codes are described in detail in De Donder \& Vanbeveren (2004). However, the theoretical treatment of binary evolution is still subject to a number of uncertainties, which are typically implemented by means of parameters. For the present discussion, two uncertain aspects of binary mass transfer, characterized in population synthesis by two parameters, are of interest. The first is the fraction of the mass lost by the donor star that is actually accreted by the gainer star. This fraction necessarily lies between zero (i.e. totally non-conservative mass transfer) and one (i.e. totally conservative mass transfer), and is characterized in population synthesis by the parameter $\beta_{\mathrm{RLOF}}{ }^{4}$ (hence $0 \leq \beta_{\mathrm{RLOF}} \leq 1$ ). If $\beta_{\mathrm{RLOF}}<1$, and thus mass is lost from the system, a second critical assumption concerns how much angular momentum is taken along from the system by this lost mass. This is determined by a parameter $\eta$ (for a formal definition, see De Donder \& Vanbeveren 2004). For the present discussion, it suffices to know that the larger $\eta$, the more angular momentum is removed from the binary by a given amount of mass loss. A common assumption made in many population synthesis studies is that the mass lost takes along only the specific orbital angular momentum of the gainer star (and thus leaves this star in a spherically symmetric way). This results in a low angular momentum loss, characterized by a low value of $\eta \sim 0.05$. An alternative assumption is that mass is lost through the second Lagrangian point and forms a circumbinary ring, taking along some of the orbital angular momentum of this ring. This entails a much larger angular momentum loss, and has been shown to result in $\eta=2.3$. Various other, less common assumptions are also possible (e.g. gainer orbital plus spin angular momentum and specific binary angular momentum), which all result in intermediate cases and can be approximated by taking $\eta=1$. The purpose of this paragraph is to investigate, for various combinations of the

4 The usual designation for this parameter is $\beta$, but we used the $\beta_{\mathrm{RLOF}}$ designation to avoid any confusion with the $\beta$ of the velocity law in the stellar winds. 
Table 12. Initial masses and orbital period, leading to a $14.8+17.2 M_{\odot}$, $2.2 \mathrm{~d}$ post-RLOF binary for different combinations of $\beta_{\mathrm{RLOF}}$ and $\eta$.

\begin{tabular}{cccc}
\hline \hline & $\beta_{\mathrm{RLOF}}=1$ & $\beta_{\mathrm{RLOF}}=0.5$ & $\beta_{\mathrm{RLOF}}=0.1$ \\
\hline$\eta=2.3$ & no solution & $34+7.9 M_{\odot}, 560 \mathrm{~d}$ & $34+15 M_{\odot}, 1000 \mathrm{~d}$ \\
$\eta=1$ & no solution & $34+7.9 M_{\odot}, 90 \mathrm{~d}$ & $34+15 M_{\odot}, 80 \mathrm{~d}$ \\
$\eta \sim 0.05$ & no solution & $34+7.9 M_{\odot}, 5.3 \mathrm{~d}$ & $34+15 M_{\odot}, 1.4 \mathrm{~d}$ \\
\hline
\end{tabular}

parameters $\beta_{\mathrm{RLOF}}$ and $\eta$, whether they can indeed result in the formation of a binary in the slow phase of case B RLOF with the physical parameters (masses and orbital period) of LSS3074, and if so, originating from which initial conditions. All calculations are performed for solar-like metallicity.

Table 12 shows the initial masses and orbital period, depending on the assumed values of $\beta_{\mathrm{RLOF}}$ and $\eta$, which produce a $14.8+17.2 M_{\odot}$ binary in the slow phase of case B RLOF and with an orbital period of 2.2 days.

Our simulations reveal that the primary progenitor was a star with initial mass $30-35 M_{\odot}$. We find no physically realistic solution if the RLOF is treated conservatively. If it is assumed that $\beta_{\mathrm{RLOF}}=0.5$, we have to start with an initial $34+7.9 M_{\odot}$ binary. It is however doubtful that a binary with such an extreme initial mass ratio would avoid merging. Our best guess is then a model with $\beta_{\text {RLOF }}<0.5$, such as the $\beta_{\text {RLOF }}=0.1$ model of Table 12 . If the matter that leaves the binary takes with it the specific angular momentum of the gainer $(\eta=0.05)$, the initial period of the binary had to be very small (of the order of 1 or 2 days) and this means that the progenitor binary first went through a case A RLOF phase followed by case B. Interestingly, our simulations show that the slow case B RLOF will stop after some $10000 \mathrm{yr}$. The primary star then will be a $11-12 M_{\odot}$ WR star, i.e. the binary will be very similar to the WR binary CQ Cep.

\subsection{Summary and conclusions}

From what we have seen above, LSS 3074 is clearly a very peculiar binary system that challenges our current methods of binary spectral analysis. In this paper, we have tried to get a deeper understanding of this system, but we encountered a number of problems that prevent us from establishing a fully consistent explanation of all the properties of the system. Here we discuss these problems and try to highlight some avenues for possible solutions.

The properties of the components of LSS 3074 that we determined from our spectral and photometric analysis do not concur with those of genuine early O-type supergiants as determined, for instance, by Bouret et al. (2012). Indeed, these latter authors used the CMFGEN code to analyse UV and optical spectra of typical early and mid-O supergiants. They found that O4-4.5 supergiants have radii between 18.5 and $21.6 R_{\odot}, \log g$ in the range 3.51 to 3.66 and $\log L / L_{\odot}$ between 5.83 and 5.96. For O67.5 supergiants, Bouret et al. (2012) determined radii between 20.5 and $21.3 R_{\odot}, \log g$ between 3.41 and 3.54 , and $\log L / L_{\odot}$ in the range 5.68 to 5.80. The components of LSS 3074 have significantly smaller radii, which are actually even smaller than the typical radii of early-O main-sequence stars. Stars as big as the supergiants analysed by Bouret et al. (2012) would not fit into such a short-period binary system as LSS 3074, even considering, as we have here, the possibility of an overcontact configuration. The luminosities of the components of LSS 3074 are also far from those determined by Bouret et al. (2012), and are again lower than those of main-sequence stars of the same spectral type. As for the $\log g$ of the stars in LSS 3074, their values are higher than those of genuine supergiants, indicating a more compact stellar atmosphere than for supergiants. The values we found are intermediate between those of giants and mainsequence stars. Last but not least, the masses of the components (14.6 and $17.2 M_{\odot}$ for the primary and secondary, respectively) are considerably lower than the spectroscopic or evolutionary masses of genuine $\mathrm{O}$ supergiants, which are around $50 M_{\odot}$ for early-O supergiants and around $40 M_{\odot}$ for mid-O supergiants (Bouret et al. 2012). In fact, the masses that we determined are more compatible with those of O9-B0 main-sequence stars.

Yet, despite these very discrepant stellar properties, the spectrum of the primary star of LSS 3074 is very similar to the spectrum of HD 16691 (O4 If) analysed by Bouret et al. (2012), including the presence of $\mathrm{N} v \lambda \lambda 4605-4620$ in absorption and the weakness of N IV $\lambda$ 4058. This situation suggests that the spectra of the LSS 3074 could be biased in some way. Our spectral analysis relies on the individual spectra reconstructed via spectral disentangling. Such a reconstructed spectrum is a brightnessweighted mean over the various parts of the stellar surface that can be observed given the inclination of the system. Owing to the effect of gravity darkening, the temperature at the surface of a rotating star is not uniform: the polar regions that have the higher value of $\log g$ are hotter than the equatorial regions where $\log g$ is lower. Things are even more complicated in a close binary system, where the local acceleration of gravity is determined by the gradient of the Roche potential $\nabla \Omega$ (e.g. Palate \& Rauw 2012; Palate et al. 2013). To evaluate whether or not this situation leads to a surface gravity that comes close to that of a supergiant, we computed $|\nabla \Omega|$ over the surface of the primary star, adopting a Roche lobe filling factor of 1.008 . We then compared this value to the acceleration of gravity one would have for a single star of same mass and radius. Except for a small region near the bridge of material that connects both stars, $|\nabla \Omega|$ is typically between 3 and 9 times larger than the $g$ value for a single star. Therefore, the Roche potential should lead to an increase of $\log g$ compared to its value if binarity is neglected. Hence, this situation cannot explain the discrepancy between the stellar properties and the observed spectrum. A possible way out could be the effect of the radiation pressure on the potential. Howarth (1997) has shown that radiation pressure leads to a scaling of the potential and a reduction of the local gravity by a factor $1-\Gamma$, where $\Gamma$ is a measure of the relative importance of radiation pressure over gravity. The primary star of LSS 3074 could have lost a substantial fraction of its initial mass to the surroundings and, as a result, appears now as a very hot object, significantly hotter than expected from its mass. This assumption is strengthened by the He and CNO primary surface abundances, as shown in Sect. 9.1. Given the high temperature of the components of LSS 3074, the resulting strong radiation pressure appears then to be the best candidate to explain why the stars display unusual spectra.

Another odd feature of LSS 3074 is the discrepancy between the brightness ratios inferred from spectroscopy and photometry. The spectroscopic brightness ratio mainly stems from the weakness of the secondary spectral lines. One possibility could be that these lines are filled in by emission either from the primary star or from circumstellar material. Some evidence for circumstellar emission can be seen in the He I $\lambda 5876$ and He II $\lambda 5412$ lines of the recontructed spectra, which are flanked by emission wings in the case of the secondary. Yet, it seems unclear whether this effect applies to all spectral lines and would be sufficient to explain the discrepancy between photometry and spectroscopy.

The fact that the light curve of LSS 3074 is actually better fitted by a simple sine function than by the NIGHTFALL model suggests that the components of the binary system might actually be more elongated than expected from the Roche potential. The 
inclusion of radiation pressure in the calculation of the surface shape can actually lead to systems that are detached whilst they would be in a contact configuration based only on the Roche potential (Drechsel et al. 1995; Palate et al. 2013). Radiation pressure also changes the ellipticity of the stars, making them appear less elongated for a given value of the polar radius (e.g. Fig. 3 Palate et al. 2013), although a further increase in the polar radius could lead to a higher ellipticity whilst preserving a detached configuration (Drechsel et al. 1995). Whilst a detailed modelling of the light curve of LSS 3074, including the effect of radiation pressure, is beyond the scope of the present study, we note that the temperature distribution at the surface of the stars will also be altered and this could possibly help explain the observed light curve. A detached configuration resulting from the effect of radiation pressure could in principle also help bring the photometric and spectroscopic brightness ratios into better agreement.

Acknowledgements. The Liège team acknowledges support from the Fonds de la Recherche Scientifique (F.R.S/FNRS) including especially an F.R.S/FNRS Research Project (T.0100.15), as well as through an ARC grant for Concerted Research Actions financed by the French Community of Belgium (WalloniaBrussels Federation), and an XMM PRODEX contract (Belspo). We are particularly grateful to Reinhold Haefner for having made his IBVS data available and to Ella Antokhina and Igor Antokhin for discussions on the photometric solution. EG is greatly indebted to Stephanie Wachter and Suzanne Tourtelotte for having spared no pains in facilitating the interaction with the YALO archives. We are grateful to I. Stevens, H. Sana, Y. Nazé, J.-M. Vreux, and M. da Silva Pires for taking some of the spectroscopic data or for discussions on earlier versions of this work. We thank John Hillier for making his code available.

\section{References}

Almeida, L. A., Sana, H., de Mink, S. E., et al. 2015, ApJ, 812, 102 Antokhina, E. A., \& Kumsiashvili, M. I. 1999, Astron. Lett., 25, 662 Antokhina, E.A., Antokhin, I.I., \& Cherepashchuk, A. M. 2013, Astron. Astrophys. Trans., 28, 3

Arnaud, K. A. 1996, in Astronomical Data Analysis Software and Systems V, eds. G. Jacoby, \& J. Barnes, ASP Conf. Ser., 101, 17

Asplund, M., Grevesse, N., Sauval, A. J., \& Scott, P. 2009, ARA\&A, 47, 481

Bohlin, R. C., Savage, B. D., \& Drake, J. F. 1978, ApJ, 224, 132

Bouret, J.-C., Hillier, D. J., Lanz, T., \& Fullerton, A. W. 2012, A\&A, 544, A67

Conti, P. S. 1973, ApJ, 179, 161

Conti, P. S. 1974, ApJ, 187, 539

Conti, P. S., \& Alschuler, W. R. 1971, ApJ, 170, 325

Conti, P. S., \& Frost, S. A. 1977, ApJ, 212, 728

Crowther, P. A., Hillier, D. J., Evans, C. J., et al. 2002, ApJ, 579, 774

Curcio, J. A., Drummeter, L. F., \& Knestrick, G. L. 1964, Appl. Opt., 3, 1401

De Donder, E., \& Vanbeveren, D. 2004, New Astron. Rev., 48, 861

de Loore, C., \& Vanbeveren, D. 1994, A\&A, 292, 463

de Mink, S. E., Pols, O. R., \& Hilditch, R. W. 2007, A\&A, 467, 1181

de Mink, S. E., Cantiello, M., Langer, N., et al. 2009, A\&A, 497, 243

Djurašević, G., Vince, I., Antokhin, I. I., et al. 2012, MNRAS, 420, 3081

Dray, L. M., \& Tout, C. A. 2007, MNRAS, 376, 61

Drechsel, H., Haas, S., Lorenz, R., \& Gayler, S. 1995, A\&A, 294, 723

Eggleton, P. P. 1983, ApJ, 268, 368

González, J. F., \& Levato, H. 2006, A\&A, 448, 283

Gosset, E., Royer, P., Rauw, G., Manfroid, J., \& Vreux, J.-M. 2001, MNRAS, 327,435

Gosset, E., Rauw, G., Manfroid, J., et al. 2005, Proceedings of Massive Stars and High-Energy Emission in OB Associations, a workshop of the JENAM 2005, Distant Worlds, held in Liège (Belgium), July 4-7, eds. G. Rauw, Y. Nazé, R. Blomme, \& E. Gosset, 77

Gray, D. F. 2008, The Observation and Analysis of Stellar Photospheres, 3rd edn. (Cambridge University Press)

Gray, R. O. 2010, http://www . appstate.edu/ grayro/spectrum/ spectrum276/node38.html

Haefner, R., Simon, K. P., \& Fiedler, A. 1994, IBVS, 3969, 1

Heger, A., Langer, N., \& Woosley, S. E. 2000, ApJ, 528, 368

Herbig, G. 1995, ARA\&A, 33, 19

Hillier, D. J., \& Miller, D. L. 1998, ApJ, 496, 407

Horne, K. 1991, in Fundamental Properties of Cataclysmic Variable Stars: 12th North American Workshop on Cataclysmic Variables and Low Mass X-ray Binaries, ed. A. W. Shafter (SDSU Press), 23
Houk, N., \& Cowley, A.P. 1975, in University of Michigan Catalogue of twodimensional spectral types for the HD stars, Vol. I. Declinations -90 to $-53^{\circ}$ Howarth, I. D. 1997, The Observatory, 117335

Hurley, J. R., Tout, C. A., \& Pols, O. R. 2002, MNRAS, 329, 897

Jansen, F., Lumb, D., Altieri, B., et al. 2001, A\&A, 365, L1

Kaufer, A., Stahl, O., Tubbesing, S., et al. 1999, The Messenger, 95, 8

Kippenhahn, R., \& Weigert, A. 1967, Z. Astrophys., 65, 251

Langer, N. 2012, ARA\&A, 50, 107

Linder, N., Rauw, G., Sana, H., De Becker, M., \& Gosset, E. 2007, A\&A, 474, 193

Linder, N., Rauw, G., Martins, F., Sana, H., De Becker, M., \& Gosset, E. 2008, A\&A, 489, 713

Lorenzo, J., Negueruela, I., Val Baker, A. K.F., et al. 2014, A\&A, 572, A110

MacConnell, D. J. 1981, A\&AS, 44, 387

Mahy, L. 2011, Ph.D. Thesis, University of Liège, Belgium

Mahy, L., Gosset, E., Sana, H., et al. 2012, A\&A, 540, A97

Maíz Apellániz, J., Sota, A., Arias, J. I., et al. 2016, ApJS, 224, 4

Manfroid, J. 1993, A\&A, 271, 714

Manfroid, J., Royer, P., Rauw, G., \& Gosset, E. 2001, in Astronomical Data Analysis Software and Systems X, eds. F. Harnden, F. Primini, \& H. Payne, ASP Conf. Ser., 238, 373

Martins, F. 2011, Bull. Soc. Roy. Sci. Liège, 80, 29

Martins, F., \& Plez, B. 2006, A\&A, 457, 637

Martins, F., Schaerer, D., \& Hillier, D. J. 2005, A\&A, 436, 1049

Martins, F., Hervé, A., Bouret, J.-C., et al. 2015, A\&A, 575, A34

Mason, B. D., Gies, D. R., Hartkopf, W. I., et al. 1998, AJ, 115, 821

Morrell, N. I., \& Niemela, V. S. 1990, in Properties of hot luminous stars, ASP Conf. Ser., 7, 57

Muijres, L. E., Vink, J. S., de Koter, A., Müller, P. E., \& Langer, N. 2012, A\&A, 537, A37

Nazé, Y. 2009, A\&A, 506, 1055

Nazé, Y., Rauw, G., Vreux, J.-M., \& De Becker, M. 2004, A\&A, 417, 667

Niemela, V.S., Cerruti, M. A., Morrell, N.I., \& Luna, H. G. 1992, in Evolutionary Processes in Interacting Binary Stars (Dordrecht Kluwer: Acad. Publ.), 505

Palate, M., \& Rauw, G. 2012, A\&A, 537, A119

Palate, M., Rauw, G., Koenigsberger, G., \& Moreno, E. 2013, A\&A, 552, A39

Pigulski, A., \& Pojmański, G. 2008, A\&A, 477, 917

Podsiadlowski, P., Joss, P. C., \& Hsu, J. J. L. 1992, ApJ, 391, 246

Prinja, R. K., Barlow, M. J., \& Howarth, I. D. 1990, ApJ, 361, 607

Raucq, F., Rauw, G., Gosset, E., et al. 2016, A\&A, 588, A10

Rauw, G. 2007, DSc Thesis, University of Liège, Belgium

Rauw, G., Crowther, P. A., Eenens, P. R. J., Manfroid, J., \& Vreux, J.-M. 2002, A\&A, 392, 563

Reed, B. C. 2003, AJ, 125, 2531

Richards, M. T., Cocking, A. S., Fisher, J. G., \& Conover, M. J. 2014, ApJ, 795, 160

Sana, H., Gosset, E., \& Rauw, G. 2006a, MNRAS, 371, 67

Sana, H., Rauw, G., Nazé, Y., Gosset, E., \& Vreux, J.-M. 2006b, MNRAS, 372, 661

Sana, H., de Mink, S. E., de Koter, A., et al. 2012, Science, 337, 444

Sana, H., Le Bouquin, J.-B., Lacour, S., et al. 2014, ApJS, 215, 15

Seidensticker, K. J. 1989, A\&AS, 79, 61

Seidensticker, K. J., \& Schmidt-Kaler, Th. 1989, A\&A, 225, 192

Simón-Díaz, S., \& Herrero, A. 2007, A\&A, 468, 1063

Smith, R. K., Brickhouse, N. S., Liedahl, D. A., \& Raymond, J.C. 2001, ApJ, 556, L91

Sota, A., Maíz Apellániz, J., Walborn, N. R., et al. 2011, ApJS, 193, 24

Sota, A., Maíz Apellániz, J., Morrell, N. I., et al. 2014, ApJS, 211, 10

Stetson, P. B. 1987, PASP, 99, 191

Strüder, L., Briel, U., Dennerl, K., et al. 2001, A\&A, 365, L18

Turner, M. J. L., Abbey, A., Arnaud, M., et al. 2001, A\&A 365, L27

Underhill, A. B. 1995, ApJS, 100, 433

Vanbeveren, D., de Loore, C., Van Rensbergen, W. 1998, A\&ARv, 9, 63

van der Hucht, K. A. 1996, in Wolf-Rayet stars in the framework of stellar evolution, Liège International Astrophysical Colloquium, 33, eds. J.-M. Vreux, A. Detal, D. Fraipont-Caro, E. Gosset, \& G. Rauw, 1

Walborn, N. R. 2001, in Eta Carinae and Other Mysterious Stars: The hidden opportunities of emission spectroscopy, eds. T. R. Gull, S. Johannson, \& K. Davidson, ASP Conf. Ser., 242, 217

Walborn, N. R., Howarth, I. D., Lennon, D. J., et al. 2002, AJ, 123, 2754

Wellstein, S., Langer, N., \& Braun, H. 2001, A\&A, 369, 939

Wilms, J., Allen, A., \& McCray, R. 2000, ApJ, 542, 914

Wilson, R. E. 1974, ApJ, 189, 319

Yaşarsoy, B., \& Yakut, K. 2013, AJ, 145, 9

Zhao, E., Qian, S., Li, L., et al. 2014, New Astron., 26, 112 


\section{Appendix A}

During the above-mentioned iterative process to derive the equivalent of global differential magnitudes (see Sect.2.2), a few stars were rejected owing to suspected variability. Two stars turned out to be significantly variable: they are analysed here below.

\section{A. $1 H D 116827 \equiv C P D-61^{\circ} 3706$}

HD 116827 is known to have $\mathrm{H} \alpha$ in emission and is thus classified as a Be star (MacConnell 1981). The $\mathrm{H} \beta$ line also presents a weak emission within the absorption profile; the spectral type is B2Ve-B5Ve as derived by Houk \& Cowley (1975). Pigulski \& Pojmański (2008) observed it in photometry as part of the ASAS-3 endeavour and reported the presence of two pulsation modes with periods $0.22429 \mathrm{~d}$ and $0.45217 \mathrm{~d}$; they concluded to a $\beta$ Cep nature for this object. We confirm the variable nature of this star and the magnitudes measured in the framework of the present work are given in Table A.2, available at the CDS. These magnitudes have been analysed using the same multi-frequency Fourier methods as referred to in the main text. For each filter, we decomposed the light curve into deterministic processes considering up to three frequencies and an additional white-noise process. The dominant frequency peaks are $v=2.312,4.455,3.349 \mathrm{~d}^{-1}$ for the $B$ bandpass (semiamplitudes 0.013, 0.012, and 0.012, respectively), $v=1.210$, 2.213 , and $4.458 \mathrm{~d}^{-1}$ for the $V$ bandpass (semi-amplitudes $0.012,0.011$, and 0.010 , respectively), $v=4.761,2.236$, and $1.235 \mathrm{~d}^{-1}$ for the $R$ bandpass (semi-amplitudes $0.012,0.011$, and 0.010, respectively), and, finally, $v=1.288,2.287$, and $4.492 \mathrm{~d}^{-1}$ for the $I$ bandpass (semi-amplitudes 0.020, 0.019, and 0.018 , respectively). Considering that the one-day aliasing remains strong in the sample, we conclude that the dominant frequencies for the different bandpasses could in some cases share the same single origin. No frequency other than the dominant frequencies seems to present some ubiquity among the various filters, indicating that we are nearing the noise level. We removed the mean magnitude from each dataset and merged the four light curves aiming at decreasing the noise. The corresponding Fourier (semi-amplitude) periodogram is given in Fig. A.1. Four main frequency peaks can be spotted

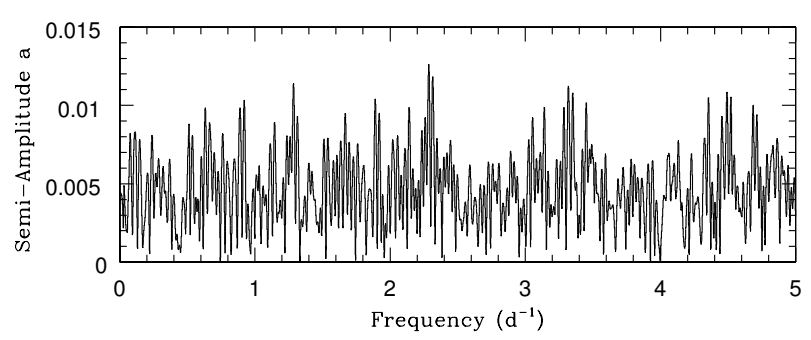

Fig. A.1. Fourier (semi-amplitude) periodogram for HD 116827.

at $v=2.286 \mathrm{~d}^{-1}$ (semi-amplitude 0.013$), v=1.286 \mathrm{~d}^{-1}$ (semiamplitude 0.011), $v=3.319 \mathrm{~d}^{-1}$ (semi-amplitude 0.011), and $v=4.355 \mathrm{~d}^{-1}$ (semi-amplitude 0.010 ). The one-day alias makes no doubt and we should be careful while making such a list. We also analysed the global data with the multi-frequency method introduced by Gosset et al. (2001). Two independent frequencies were found to be $v=2.286 \mathrm{~d}^{-1}$ (semi-amplitude 0.014 ) and $v=4.493 \mathrm{~d}^{-1}$ (semi-amplitude 0.014 ), but no other significant frequency can be spotted. The two derived independent frequencies are strongly reminiscent of those reported on the basis of the ASAS-3 photometry $\left(v=4.459 \mathrm{~d}^{-1}\right.$ and $\left.v=2.212 \mathrm{~d}^{-1}\right)$, particularly taking into account the $0.03 \mathrm{~d}^{-1}$ aliasing.

\section{A.2 LSS3072 $\equiv C P D-61^{\circ} 3692$}

This object appears in the catalogue of OB stars of Reed (2003) and is classified as O9. The magnitudes measured in the framework of the present work are given in Table A.3, available at the CDS. It is clearly variable and the light curve is plotted against time in Fig. A.2. A search for periods results in the detection of the possible frequency $v=0.1092 \mathrm{~d}^{-1}(P=9.16 \mathrm{~d})$ for each of the four bandpasses and for the combined data (semi-amplitude $0.023 \mathrm{mag}$ in $B, 0.023$ in $V, 0.024$ in $R$ and 0.023 in $I$ ). Two bumps are clearly present in Fig. A.2. A few discrepant data points are present at HJD 2451999.71 and at HJD 2452 027.54. The deletion of these data points does not change the result of the period search. However, it is worth noticing that they are separated by some three entire cycles and could thus be related to the possible main period. We have no particular reason to reject these possible outliers. 
F. Raucq et al.: Signatures of past mass exchange in LSS 3074
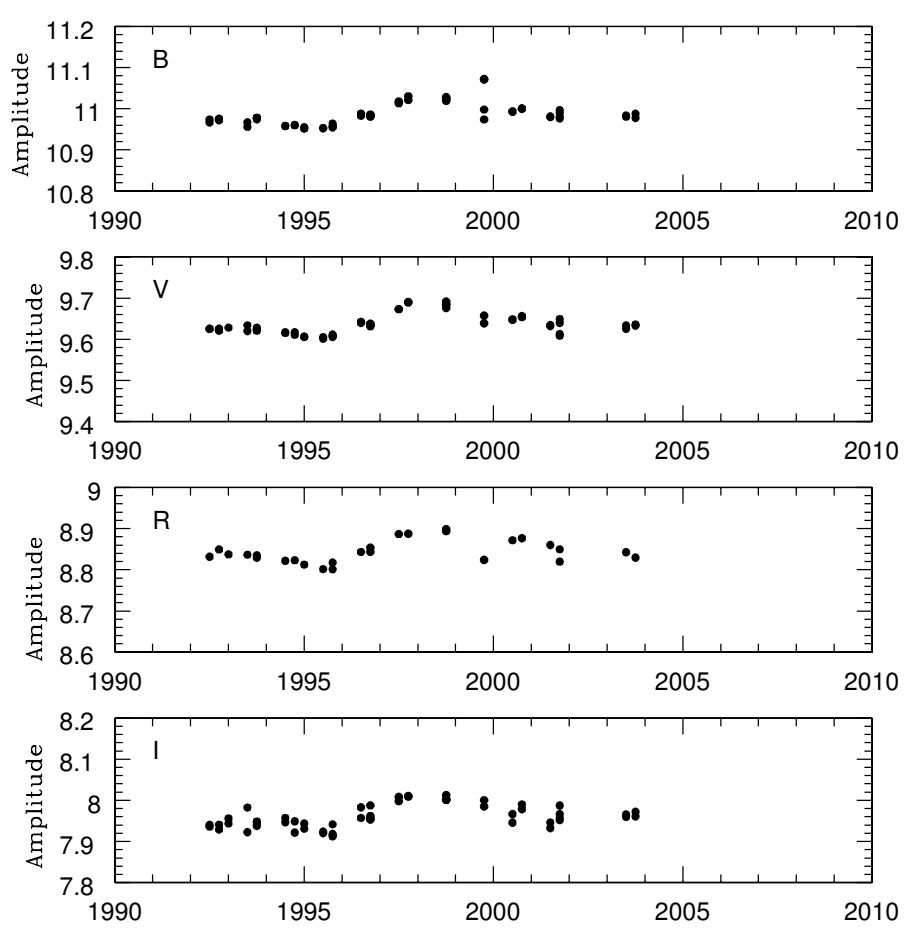

Hel. Julian Date (2450000+)
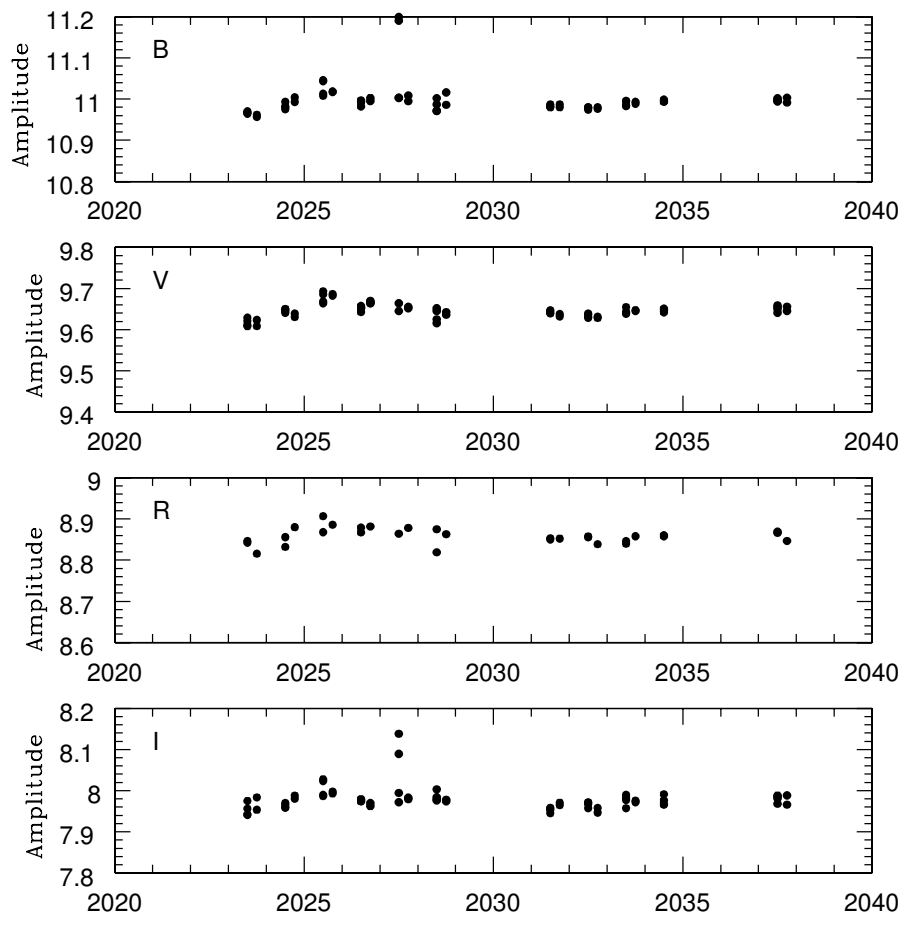

Hel. Julian Date (2450000+)

Fig. A.2. Light curve of LSS 3072. 